\title{
Solute Trapping and Solute Drag in a Phase-Field Modiel of Rapid Solidification
}

\section{N. A. Ahmad}

A. A. Wheeler

Faculty of Mathematical Studies

University of Southampton

Southampton, S017, 1Bj, UK

\section{W. J. Boettinger \\ G. B. McFadden}

U.S. DEPARTMENT OF COMMERCE

Technology Administration

National Institute of Standards

and Technology

Gaithersburg, MD 20899-0001

QC

100

.056

N0.6156 


\section{NISTIR 6156}

\section{Solute Trapping and Solute Drag in a Phase-Field Model of Rapid Solidification}

\section{N. A. Ahmad \\ A. A. Wheeler}

Faculty of Mathematical Studies

University of Southampton

Southampton, S017, 1BJ, UK

\section{W. J. Boettinger}

G. B. McFadden

U.S. DEPARTMENT OF COMMERCE

Technology Administration

National Institute of Standards

and Technology

Gaithersburg, MD 20899-0001



U.S. DEPARTMENT OF COMMERCE

William M. Daley, Secretary

TECHNOLOGY ADMINISTRATION

Gary R. Bachula, Acting Under Secretary for Technology

NATIONAL INSTITUTE OF STANDARDS

AND TECHNOLOGY

Raymond G. Kammer, Director 



\title{
Solute Trapping and Solute Drag
}

\section{in a Phase-Field Model of Rapid Solidification}

\author{
N. A. Ahmad and A. A. Wheeler \\ Faculty of Mathematical Studies \\ University of Southampton \\ Southampton, SO17 1BJ, UK \\ and \\ W. J. Boettinger and G. B. McFadden \\ National Institute of Standards and Technology \\ Gaithersburg, MD 20899, USA
}

\begin{abstract}
During rapid solidification, solute may be incorporated into the solid phase at a concentration significantly different than that predicted by equilibrium thermodynamics. This process, known as solute trapping, leads to a progressive reduction in the concentration change across the interface as the solidification rate increases. Theoretical treatments of rapid solidification using traditional sharp-interface descriptions require the introduction of separately-derived non-equilibrium models for the behavior of the interfacial temperature and solute concentrations. In contrast, phase-field models employ a diffuse-interface description, and eliminate the need to specify interfacial conditions separately. While at low solidification rates equilibrium behavior is recovered, at high solidification rates non-equilibrium effects naturally emerge from these models. In particular, in a previous study we proposed a phase-field model of a binary alloy [Wheeler et al., Phys. Rev. E 47 (1993) 1893] in which we demonstrated solute trapping. Here we show that solute trapping is also possible in a simpler diffuse interface model. We show that solute trapping occurs when the solute diffusion length, $D_{I} / V$, is comparable to the diffuse interface thickness. Here $V$ is the interface velocity, and $D_{I}$ characterizes the solute diffusivity in the interfacial region. We characterize the dependence of the critical speed for solute trapping on the equilibrium partition coefficient $k_{E}$ that shows good agreement with experiments by Aziz and coworkers [see Aziz, Metall. Mat. Trans. 27A (1996) 671]. We also show that in the phase-field model, there is a dissipation of energy in the interface region resulting in a solute drag, which we quantify by determining the relationship between the interface temperature and velocity.
\end{abstract}




\section{Introduction}

Sharp interface models of alloy solidification employ the solution to the conventional diffusion equations for heat and solute in the bulk phases. The matching of solutions at the solid-liquid interface is obtained (a) from the flux conditions required for conservation and (b) through constitutive laws for the interface temperature and the jump in concentration across the interface as functions of velocity. The latter are obtained from separately-derived models of the solute diffusion across the atomic layers associated with the interface; see, for example, the continuous growth (CG) model of Aziz and coworkers $[1,2,3]$ as well as others $[4,5,6,7,8,9]$. The velocity dependence of the jump in concentration is termed solute trapping and provides a mechanism whereby the jump vanishes at high rates of solidification in a manner consistent with experimental observations (partitionless solidification).

While this modeling approach has met with considerable success, it is clear that at high rates of solidification $V$ (around $1 \mathrm{~m} / \mathrm{s}$ ), the diffusion length $D_{L} / V$, where $D_{L}$ is the diffusion coefficient in the liquid, that is predicted by the conventional diffusion equation is comparable to the interface thickness for metals. At such length scales diffuse interface theories (see, e.g., Cahn \& Allen [10, 11] and Cahn \& Hilliard [12]) are often found to provide more reasonable descriptions of the diffusion process in and around the interfacial region. The phase-field model presented in this paper provides a common framework for modeling both the bulk phases and the interfacial region, and for avoiding the requirement for separately-derived constitutive laws for the interface conditions. The composition profile through the interfacial region, as well as in the bulk phases, is obtained through this method.

Many of the ingredients of the phase-field approach to solute trapping can be found in the continuum interface models of Baker \& Cahn $[13,14,15]$ and Hillert \& Sundman [16] (for solid state transformations). They compute the velocity-dependence of the concentration profile across a moving diffuse phase boundary for a prescribed chemical potential profile. The latter also make a separate analysis of the free energy available for, and that dissipated by, the boundary motion. The energy dissipated is called solute drag, a subject also treated by Cahn [17]. As we will see in this paper, solute trapping and solute drag are included in the phase-field governing equations that arise naturally out of gradient flow thermodynamics.

The phase-field model for modeling solidification uses a scalar variable $\phi$ (the phase field) to describe the thermodynamic state (liquid or solid) of the various regions of a system. Interfacial regions between liquid and solid are identified by smooth but highly localized transitions of the phase-field variable. For numerical calculations, the advantage of the phase-field formulation of solidification is that the interface 
is not tracked but is given implicitly by a contour of the variable $\phi$. Phase-field models of solidification for pure materials have existed for many years $[18,19,20]$. With recent advances in supercomputing this approach has allowed the computation of realistic complicated growth morphologies such as dendritic growth $[21,22,23,24,25,26,27]$.

For alloys, a model for diffuse interface motion in a system with a miscibility gap in a solid solution phase has been treated by Langer \& Sekerka [28]. However, only recently has the phase-field method been extended to alloy solidification. Application to alloy solidification was performed for an isothermal binary alloy by Wheeler, Boettinger, \& McFadden [29] (WBM1), who also studied its properties in the sharp interface limit. Löwen, Bechoefer, \& Tuckerman [30] also discuss the formal analogy between an isothermal binary alloy phase-field model and the non-isothermal phase-field model for a pure material. Caginalp \& Xie [31] described a phase-field model of a non-isothermal binary alloy. They studied a variety of different sharp interface limits and recovered versions of the equilibrium conditions at a sharp interface, none of which exhibited solute trapping. Lin \& Rogers [32] have also studied an order parameter model for a binary liquid that is based on the general framework developed by Fried \& Gurtin $[33,34]$ for order parameter models that describe configurational forces [35]. Realistic simulations of alloy dendritic growth have been performed by Warren \& Boettinger [36]. Phase-field models have also recently been developed for eutectic alloys, by a number of workers $[37,38,39,40,41]$.

The phase-field model of a binary alloy in WBM1 is based on a single gradient energy term in the phase-field variable $\phi$ and constant solute diffusivity. The phase-field then varies through the interfacial region on a length scale $\ell_{\epsilon}$ that is associated with the gradient energy coefficient $\epsilon$. We examined the sharp interface limit in which $\ell_{\epsilon}$ is much smaller than the diffusion length $D_{L} / V$, and recovered the conventional sharp-interface jump conditions based on local equilibrium assumptions. In particular, solute trapping was not found to be possible in this limit. In subsequent work [42] (WBM2), we developed a phase-field model of solute trapping in a binary alloy which included gradient energy terms in $\phi$ and the solute concentration $c$. In the WBM2 model, the phase field and solute field have independent length scales $\ell_{\epsilon}$ and $\ell_{\delta}$, respectively, in the interfacial region, that are associated with the corresponding gradient energy coefficients $\epsilon$ and $\delta$. We considered a limiting situation $\ell_{\epsilon} \ll \ell_{\delta}$, and demonstrated that in the resulting model solute trapping occurs when the diffusion length $D_{L} / V$ becomes comparable to $\ell_{\delta}$. WBM1 and WBM2 both considered the case of equal solid and liquid diffusivities.

In this paper we reconsider our first model (WBM1) and show that solute trapping is indeed predicted, but in a different limit in which the interface thickness remains finite but the interface 
velocity is large enough that the solute diffusion length is comparable to $\ell_{\epsilon}$ (first briefly reported in [43], and described in detail in [44]). In particular, solute trapping can be recovered without the necessity of introducing a solute gradient energy term. Because we now also include the possibility of a variable diffusion coefficient through the interface, the relevant diffusion length is $D_{I} / V$, where $D_{I}$ is a measure of the interface diffusivity. We are able to relate our results to the Aziz CG theory; in particular, we obtain a prediction for the dependence of the characteristic trapping velocity on the equilibrium partition coefficient which is in good agreement with recent experiments [45].

Conti $[46,47]$ has extended the WMB2 model to include nonisothermal and time-dependent effects and found that the transient solute segregation at the interface can differ significantly from the predictions of the CG model. Fife \& Charach [48] have studied a number of different sharp interface limits for a class of phase-field models of a binary alloy which include WBM2 as a special case. The solute trapping they observe is related to the solute gradient energy as in WBM2. Plapp \& Gouyet [49] have considered mean-field equations derived from lattice gas models, and examined numerically the isothermal dynamics of planar solidification; they observe oscillations of the growth velocity during solidification.

Conti [50] and Kim, Kim, \& Suzuki [51] have also independently considered solute trapping in the context of the WBM1 model, with similar conclusions concerning the mechanism of solute trapping based on numerical computations. Conti has extended the model to include nonisothermal effects and computed one-dimensional, time-dependent solutions for planar growth that show good agreement with predictions of the CG model. Kim, Kim, \& Suzuki derive an approximate analysis for the effect of trapping at low velocities, and obtain good agreement with low-velocity numerical calculations.

The outline of this.paper is as follows. In Section 2 we describe the general characteristics of existing solute trapping and solute drag models in our notation and compare them in a general way to the phase-field approach. In Section 3 we summarize the details of the phase-field model that we consider. Section 4 presents numerical calculations for one-dimensional solutions of the phase-field equations that exhibit solute trapping at high solidification rates. Some asymptotic results in the highvelocity limit are given in Section 5 that reinforce the numerical results and provide explicit expressions for the characteristic velocity at which solute trapping becomes important. A discussion is provided in Section 6, and conclusions appear in Section 7 . 


\section{Overview of Trapping and Phase-Field Models}

\subsection{Trapping Models}

Binary alloy solute trapping models are based on an analysis of diffusional jumps across the interface. For this purpose the driving forces for diffusion and crystallization are required. We consider free energy densities in the liquid and solid phases, which we denote by $f_{L}\left(c_{L}, T\right)$ and $f_{S}\left(c_{S}, T\right)$, respectively, where $T, c_{L}$, and $c_{S}$ represent the temperature and the concentrations (mole fractions) of species $B$ in the liquid and solid phases at the liquid-solid interface. ${ }^{1}$

The chemical potentials of species $A$ and $B$ in each phase are given by

$$
\begin{gathered}
\mu_{A}^{L}=f_{L}\left(c_{L}, T\right)-c_{L} \frac{\partial f_{L}}{\partial c_{L}}\left(c_{L}, T\right), \\
\mu_{B}^{L}=f_{L}\left(c_{L}, T\right)+\left(1-c_{L}\right) \frac{\partial f_{L}}{\partial c_{L}}\left(c_{L}, T\right), \\
\mu_{A}^{S}=f_{S}\left(c_{S}, T\right)-c_{S} \frac{\partial f_{S}}{\partial c_{S}}\left(c_{S}, T\right), \\
\mu_{B}^{S}=f_{S}\left(c_{S}, T\right)+\left(1-c_{S}\right) \frac{\partial f_{S}}{\partial c_{S}}\left(c_{S}, T\right) .
\end{gathered}
$$

Thermodynamic equilibrium at the interface is expressed by equality of the chemical potentials, $\mu_{A}^{L}=$ $\mu_{A}^{S}$ and $\mu_{B}^{L}=\mu_{B}^{S}$, which can be represented graphically by a common tangent construction in $f$-c space at fixed $T$. Equivalent conditions are often expressed in terms of the interdiffusion potentials $\mu^{L}=\mu_{B}^{L}-\mu_{A}^{L}=\partial f_{L} / \partial c_{L}$ and $\mu^{S}=\mu_{B}^{S}-\mu_{A}^{S}=\partial f_{S} / \partial c_{S}$ in each phase, and the free energy change on solidification, $\Delta F_{S}$, defined by

$$
\begin{gathered}
\Delta F_{S}=f_{S}\left(c_{S}, T\right)-\left\{f_{L}\left(c_{L}, T\right)+\left(c_{S}-c_{L}\right) \frac{\partial f_{L}}{\partial c_{L}}\left(c_{L}, T\right)\right\} \\
=\left(1-c_{S}\right)\left\{\mu_{A}^{S}\left(c_{S}, T\right)-\mu_{A}^{L}\left(c_{L}, T\right)\right\}+c_{S}\left\{\mu_{B}^{S}\left(c_{S}, T\right)-\mu_{B}^{L}\left(c_{L}, T\right)\right\} .
\end{gathered}
$$

This expression represents the free energy change per unit volume associated with removing material of composition $c_{S}$ from the liquid and adding it to the solid phase, and is known as the tangent to curve rule [14]. Equivalent equilibrium conditions are then given by

$$
\mu^{L}-\mu^{S}=0, \quad \Delta F_{S}=0 .
$$

\footnotetext{
${ }^{1}$ For simplicity we assume a constant molar volume $v_{m}$ in the system, which eliminates possible convective effects, such as those associated with a density change on solidification. The free energies and their associated chemical potentials are measured in units of energy per unit volume.
} 
In the dilute solution limit, the equation $\mu^{L}-\mu^{S}=0$ results in the expression $c_{S}=k_{E} c_{L}$ that defines the equilibrium partition coefficient $k_{E}$ in terms of the free energies, and the equation $\Delta F_{S}=0$ results in an expression for the liquidus temperature $T=T_{M}+m_{L} c_{L}$, that defines the liquidus slope $m_{L}$ in terms of the free energies, where $T_{M}$ is the pure solvent melting point. Under non-equilibrium conditions, nonzero values of the quantity $\mu^{L}-\mu^{S}$ and the free energy change on solidification $\Delta F_{S}$ are interpreted as driving forces for solute distribution and phase change, respectively. These lead to response functions [14] that provide non-equilibrium relations between $c_{L}, c_{S}, T$, and the interface velocity $V$. The response functions define kinetic laws that reduce to the above equilibrium conditions (6) when $V=0$.

\subsubsection{Expressions for the Partition Coefficient}

A simple example of a non-equilibrium model for solute segregation can be obtained by assuming that the jump in interdiffusion potential actually occurs over a small interface width $\ell$, typically of atomic dimensions, to create a gradient $\nabla \mu \approx\left(\mu^{L}-\mu^{S}\right) / \ell$ and a diffusion flux $J$ of solute across the interface, measured in the reference frame of the sample, as

$$
J=\frac{-M_{0}}{v_{m}} c_{L}\left(1-c_{L}\right) \frac{\left(\mu^{L}-\mu^{S}\right)}{\ell}
$$

here we have arbitrarily inserted a "thermodynamic factor" of $c_{L}\left(1-c_{L}\right)$ in order to simplify the resulting expressions below, while retaining generality by allowing for a possibly concentration-dependent mobility coefficient $M_{0}$. For steady-state growth at velocity $V$, the flux must also satisfy a solute balance law,

$$
J=\frac{V}{v_{m}}\left(c_{L}-c_{S}\right),
$$

where $v_{m}$ is the molar volume. Combining these expressions gives

$$
\mu^{L}-\mu^{S}=-V \frac{\left(c_{L}-c_{S}\right) \ell}{M_{0} c_{L}\left(1-c_{L}\right)},
$$

which in the dilute solution limit leads to a relation for the partition coefficient $k=c_{S} / c_{L}$ of the form

$$
\ln \left(k / k_{E}\right)=\frac{V}{V_{D}}(1-k)
$$

where $V_{D}=D_{I} / \ell$ is a characteristic trapping velocity with an interface diffusivity $D_{I}=M_{0} R T / v_{m}$, and $R$ is the universal gas constant. This expression exhibits the low velocity limit $k \approx k_{E}$ for $V / V_{D} \ll 1$ and the high velocity limit $k \approx 1$ for $V / V_{D} \gg 1$.

Baker \& Cahn [14] and Hillert \& Sundman [16] analyze diffusion through the interface with continuum models that make assumptions about the details of the variation of the interdiffusion potentials 
and the diffusion coefficient through the interface. In contrast, the CG model of Aziz is based on a model of forward and reverse fluxes across the interfacial region using chemical reaction theory such that $J$ is nonlinear in the quantity $\mu^{S}-\mu^{L}$. We note that linearization of their expression for $J$ leads to a prefactor of $c_{S}\left(1-c_{L}\right)$ in Eq. (7). In the CG model the partition coefficient $k$ depends on the interface velocity through an expression of the form

$$
k=\frac{k_{E}+V / V_{D}}{1+V / V_{D}}
$$

in the dilute solution limit for $k_{E}<1$. Here $V_{D}$ represents the characteristic interface velocity scale on which the solute trapping occurs. The quantity $V_{D}$ is given by $D_{I} / \ell$ where $D_{I}$ represents an interfacial diffusivity and $\ell$ is the thickness of the interface, equal to the atomic jump distance. Since, however, $D_{I}$ is not subject to direct experimental determination, the value of $V_{D}$ cannot be measured directly. Instead, values for $V_{D}$ are inferred by fitting the observed dependence of $k$ on $V$ using the expression (11). By studying trapping in a number of alloys, Smith \& Aziz [45] have found that the trapping velocities $V_{D}$ depend on the particular alloy. In particular, $V_{D}$ was found to correlate most strongly with the equilibrium partition coefficient $k_{E} ; V_{D}$ was not found to correlate strongly with either the liquid diffusivity $D_{L}$ or the solid diffusivity $D_{S}$. Smith \& Aziz [45] also consider a multi-step CG formulation in which the interface consists of a finite number of discrete layers that, in the proper limit, resembles a diffuse interface.

The Aziz CG model has been extended to $k_{E}>1$ [52]. The appropriate expression for $k$ can also be derived using the formalism of Ref. [53] to give

$$
1 / k=\frac{1 / k_{E}+V / V_{D}}{1+V / V_{D}}
$$

in the dilute solution limit. This form is similar to that of (11), but involves instead the reciprocals of $k$ and $k_{E}$. The expressions (11) and (12) are based on a redistribution potential diagram in which a barrier height is assumed to maintain a fixed distance above the higher of the two double-well minima (see [53]). Most of the available experimental data pertain to the case $k_{E}<1$, although in this paper we shall also briefly consider the case $k_{E}>1$ for completeness.

Note that the CG expression (11) for $k_{E}<1$ can be expressed in the form

$$
k-k_{E}=\frac{V}{V_{D}}(1-k)
$$

which exhibits the same high and low velocity limits as the expression (10). Indeed the two become identical when $k_{E}$ tends to unity. 


\subsubsection{Expressions for the Interface Temperature}

Expressions for the interface temperature can be derived from models involving the change in free energy density upon solidification, $\Delta F_{S}$. For example, a model that relates the interface velocity to the driving force $\Delta F_{S}[54]$ is given by

$$
V=V_{c}\left[1-\exp \left(\frac{v_{m} \Delta F_{S}}{R T}\right)\right] \approx-V_{c} \frac{v_{m} \Delta F_{S}}{R T}
$$

where $V_{c}$ is the maximum speed of crystal growth at infinite driving force. Such a model assumes that the entire free energy change upon solidification can be devoted to driving the solidification. So-called solute drag models assume that a fraction of $\Delta F_{S}$ is dissipated at the interface; the amount dissipated is denoted by $\Delta F_{D}$. The driving force for solidification is then assumed to be provided by the remainder, denoted by $\Delta F_{C}=\Delta F_{S}-\Delta F_{D}$, leading to an alternate expression of the form

$$
V=V_{c}\left[1-\exp \left(\frac{v_{m} \Delta F_{C}}{R T}\right)\right] \approx-V_{c} \frac{v_{m}\left(\Delta F_{S}-\Delta F_{D}\right)}{R T} .
$$

Aziz \& Kaplan [3] consider a specific model for the interface dissipation given by

$$
\Delta F_{D}=\left(c_{L}-c_{S}\right)\left\{\left[\mu_{A}^{S}\left(c_{S}, T\right)-\mu_{A}^{L}\left(c_{L}, T\right)\right]\left[\mu_{B}^{S}\left(c_{S}, T\right)-\mu_{B}^{L}\left(c_{L}, T\right)\right]\right\}
$$

In both cases an expression for the interface temperature of the form

$$
T=T_{M}+\frac{m_{L} c_{\infty}}{k}\left(\frac{1-k+[k+(1-k) \alpha] \ln \left(k / k_{E}\right)}{1-k_{E}}\right)-\frac{V}{\tilde{\mu}}
$$

can be derived in the dilute solution limit [54]. Here $\alpha=1$ if solute drag is included and $\alpha=0$ if solute drag is negligible. The parameter $\alpha$ may also be interpreted as a measure of the concentration of the material transferred from the liquid to the solid state [54]. The equilibrium liquidus slope is $m_{L}$, and the kinetic coefficient $\tilde{\mu}$ is given by

$$
\tilde{\mu}=\frac{V_{c} L}{R T_{M}^{2}}
$$

in these dilute models, where $L$ is the latent heat per mole of the pure solvent.

It is useful to consider in more detail the solute drag. The energy dissipated per unit volume per unit time due to diffusion through the interfacial region is obtained by considering the term

$$
\mathcal{D}=\frac{1}{v_{m}} \vec{J} \cdot \nabla \mu
$$

across the interface. For steady-state solidification, the energy dissipated per unit volume solidified is given by $[16,3]$

$$
\mathcal{P}=\frac{v_{m}}{V} \int_{-\infty}^{\infty} J \cdot \frac{d \mu}{d x} d x
$$


$\Delta F_{D}$ as given by Eq. (16) is an approximation to Eq. (20) (see Aziz \& Kaplan [3]). Ågren [55] has proposed a model for $\Delta F_{D}$ where $c_{L}$ in the prefactor in Eq. (16) is replaced by the mean of $c_{L}$ and $c_{S}$. This yields a value of $\alpha=1 / 2$ in the expression (17). Hillert \& Sundman evaluate the solute drag directly using the solutions to their diffusion equations [16]. Gurtin and Voorhees [56] develop a general thermodynamic description of a sharp interface far from equilibrium which includes discussion of forces, fluxes, and solute drag. Liu [57] provides a compact summary of solute drag models for solidification.

\subsection{The Phase-Field Model}

The WBM1 model [29] is based on the Helmholtz free energy functional given by

$$
\mathcal{F}=\int_{\Omega}\left[f(\phi, c, T)+\frac{\epsilon^{2}}{2}|\nabla \phi|^{2}\right] d V
$$

where $\Omega$ is the volume occupied by the system and the gradient energy coefficient $\epsilon^{2}$ is assumed to be constant. The phase-field variable $\phi$ vanishes in the liquid phase, and is unity in the solid phase. In the phase-field model for a binary alloy, the free energy density $f(\phi, c, T)$ is based on the bulk free energies $f_{L}$ and $f_{S}$ and can be written in the form

$$
f(c, T, \phi)=p(\phi) f_{S}(c, T)+\{1-p(\phi)\} f_{L}(c, T)+\frac{W(c)}{4} g(\phi),
$$

where

$$
g(\phi)=\phi^{2}(1-\phi)^{2}, \quad p(\phi)=\phi^{2}(3-2 \phi)
$$

$g(\phi)$ is a double well with minima at $\phi=0$ and $\phi=1$, and $p(\phi)$ satisfies $p(0)=0$ and $p(1)=1 . W(c)$ represents a barrier height that is related to the surface energy and interface thickness [29]. In either of the bulk phases, $g(\phi)$ vanishes and $f(\phi, c, T)$ reduces to the bulk free energy density $f_{L}$ or $f_{S}$.

The governing equations are chosen to ensure that the Helmholtz free-energy functional $\mathcal{F}$ is monotonicly decreasing in time, and to conserve the total solute within the system, by putting

$$
\begin{gathered}
\frac{\partial \phi}{\partial t}=-M_{1} \frac{\delta \mathcal{F}}{\delta \phi} \\
\frac{\partial c}{\partial t}=\nabla \cdot M_{2}\left(c(1-c) \nabla \frac{\partial f}{\partial c}\right),
\end{gathered}
$$

where $M_{1}>0$ and $M_{2}>0$. The constant $M_{1}$ is related to the interface kinetic coefficient (see Eq. (48) below), and the solute mobility coefficient $M_{2}$ is related to the solute diffusivity $D$ (see Eq. (49) below). 
We now cast these governing equations in a different form to enable comparison to existing trapping models. In a frame moving with velocity $V$, the steady-state 1-D phase-field equations have the form

$$
\begin{aligned}
& \frac{-V}{M_{1}} \frac{d \phi}{d z}=\epsilon^{2} \frac{d^{2} \phi}{d z^{2}}-\frac{\partial f}{\partial \phi}(\phi, c, T) \\
& \frac{-V}{v_{m}} \frac{d c}{d z}=-\frac{d J}{d z}
\end{aligned}
$$

and the flux $J$ is defined in terms of the interdiffusion potential $\mu=\partial f / \partial c$ by

$$
v_{m} J=-c(1-c) M_{2} \frac{d \mu}{d z}
$$

We assume the interfacial layer extends over the range $-\ell / 2<z<\ell / 2$, where to a good approximation the solute field varies from $c_{S}$ to $c_{L}$ and the phase field assumes its far-field values.

\subsubsection{Driving Force for Solute Redistribution}

The solute equation (27) can be integrated once to yield

$$
\frac{V}{v_{m}}\left(c-c_{S}\right)=J
$$

where we have assumed that the flux vanishes in the solid where $c=c_{S}$. Using the definition (28), this expression can be manipulated to obtain

$$
\frac{d \mu}{d z}=\frac{-v_{m} J}{c(1-c) M_{2}}=\frac{-V\left(c-c_{S}\right)}{c(1-c) M_{2}}
$$

and integrating over the layer gives

$$
\mu\left(c_{L}\right)-\mu\left(c_{S}\right)=-V \int_{-\ell / 2}^{+\ell / 2} \frac{\left(c-c_{S}\right)}{c(1-c) M_{2}} d z .
$$

This expression has the from of a response function analogous to Eq. (9) that relates the driving force for solute redistribution, $\mu\left(c_{L}\right)-\mu\left(c_{S}\right)$, to the interface velocity, with a concentration-dependent factor given by the integral over the interfacial region. Evaluation of Eq. (31) ultimately leads to the velocity dependence of the partition coefficient $k(V)$.

\subsubsection{Driving Force for Phase Change}

Next we multiply the phase-field equation (26) by $d \phi / d z$ and integrate to obtain

$$
\frac{-V}{M_{1}} \int_{-\ell / 2}^{+\ell / 2} \phi_{z}^{2} d z=\frac{\epsilon}{2}\left[\phi_{z}^{2}\right]_{-\ell / 2}^{\ell / 2}-\int_{-\ell / 2}^{+\ell / 2} f_{\phi} \phi_{z} d z
$$


We then integrate by parts twice to obtain

$$
\begin{gathered}
\int_{-\ell / 2}^{+\ell / 2} f_{\phi} \phi_{z} d z=[f(\phi, c)]_{-\ell / 2}^{\ell / 2}-\int_{-\ell / 2}^{+\ell / 2} f_{c} c_{z} d z=[f(\phi, c)]_{-\ell / 2}^{\ell / 2}-\int_{-\ell / 2}^{+\ell / 2} f_{c}\left(c-c_{S}\right)_{z} d z \\
=\left[f-\left(c-c_{S}\right) f_{c}\right]_{-\ell / 2}^{\ell / 2}+\int_{-\ell / 2}^{+\ell / 2}\left(c-c_{S}\right) \mu_{z} d z,
\end{gathered}
$$

where we have written $\mu=\partial f / \partial c$. The latter integral may be rewritten using Eq. (29) to give

$$
\int_{-\ell / 2}^{+\ell / 2} f_{\phi} \phi_{z} d z=\left[f-\left(c-c_{S}\right) f_{c}\right]_{-\ell / 2}^{\ell / 2}+\frac{v_{m}}{V} \int_{-\ell / 2}^{+\ell / 2} J \mu_{z} d z
$$

If we set

$$
a=\int_{-\ell / 2}^{+\ell / 2} \phi_{z}^{2} d z \approx \int_{-\infty}^{\infty} \phi_{z}^{2} d z \approx \text { constant }
$$

and

$$
\frac{\epsilon}{2}\left[\phi_{z}^{2}\right]_{-\ell / 2}^{\ell / 2} \approx 0
$$

we obtain

$$
\frac{a V}{M_{1}}=-\left\{\Delta F_{S}-\frac{v_{m}}{V} \int_{-\ell / 2}^{+\ell / 2} J \mu_{z} d z\right\}
$$

where

$$
\Delta F_{S}=f\left(c_{S}\right)-\left[f\left(c_{L}\right)+\left(c_{S}-c_{L}\right) f_{c}\left(c_{L}\right)\right]
$$

is the free energy change upon solidification. This expression has the same form as Eq. (15), with a dissipation term of the form (20).

We anticipate therefore that analysis of solute trapping with the phase-field model will closely parallel results obtained by previous models. The approximations and/or assumptions made in previous trapping models regarding the behavior of the interdiffusion potential across the interface arise in a natural way from the underlying formulation of the phase-field model. Similar expressions for the difference in chemical potentials across the interface and free energy change upon solidification have been derived for the WBM1 model independently by Kim, Kim, \& Suzuki [51]. We next describe the phase-field model in more detail.

\section{Identification of Material Parameters}

The Helmholtz free-energy volume density $f(\phi, c ; T)$ in Eqn. (22) can be written in the equivalent form

$$
f(\phi, c, T)=c f_{B}(\phi, T)+(1-c) f_{A}(\phi, T)+\frac{R T}{v_{m}} I(c)
$$


where the function $I(c)=c \ln c+(1-c) \ln (1-c)$ is related to the entropy of mixing. The functions $f_{A}(\phi, T)$ and $f_{B}(\phi, T)$ represent the free energies of the pure materials $A$ and $B$ respectively with corresponding freezing temperatures $T_{A}$ and $T_{B}$, which we assume satisfy $T_{B}<T_{A}$. They are represented by double-well potentials with respect to $\phi$, as used by Kobayashi [21],

$$
f_{j}(\phi, T)=W_{j} \int_{0}^{\phi} u[u-1]\left[u-1 / 2-\beta_{j}(T)\right] d u=\frac{W_{j}}{4} g(\phi)+\frac{W_{j} \beta_{j}(T)}{6} p(\phi)
$$

for $j=A, B$, where

$$
g(\phi)=\phi^{2}(1-\phi)^{2}, \quad p(\phi)=\phi^{2}(3-2 \phi)
$$

note that $g(\phi)$ is a double well with minima at $\phi=0$ and $\phi=1$, and $p(\phi)$ satisfies $p(0)=0$ and $p(1)=1$. Here $W_{A}$ and $W_{B}$ are constants, and the temperature $T$ is a parameter in this isothermal situation. We assume that $T_{B}<T<T_{A}$, and that $-1 / 2<\beta_{A}(T)<0<\beta_{B}(T)<1 / 2$. The constants $W_{A}$ and $W_{B}$ may be related to the surface energies, $\sigma_{A}$ and $\sigma_{B}$, and the interface thicknesses, $\ell_{A}$ and $\ell_{B}$, for the pure components [29], e.g.,

$$
\sigma_{A}=\frac{\epsilon \sqrt{W_{A} / 2}}{6}, \quad \ell_{A}=\frac{\epsilon}{\sqrt{W_{A} / 2}}
$$

or

$$
\epsilon^{2}=6 \sigma_{A} \ell_{A}, \quad W_{A}=12 \sigma_{A} / \ell_{A}
$$

and the functions $\beta_{A}(T)$ and $\beta_{B}(T)$ may be related to the latent heats, e.g.

$$
\frac{W_{A} \beta_{A}(T)}{6}=L_{A} \frac{\left(T-T_{A}\right)}{T_{A}}
$$

where $L_{A}$ is the latent heat per unit volume of pure component A. Note that since we assume a constant value for $\epsilon$, we have the constraint $\sigma_{A} \ell_{A}=\sigma_{B} \ell_{B}$; in particular, assuming in addition that any one of the equalities $W_{A}=W_{B}, \sigma_{A}=\sigma_{B}$, or $\ell_{A}=\ell_{B}$ holds implies that the other two hold as well.

For the case of pure $A$, the one-dimensional phase-field equation

$$
\frac{1}{M_{1}} \frac{\partial \phi}{\partial t}=\epsilon^{2} \frac{\partial^{2} \phi}{\partial z^{2}}-f_{\phi}
$$

has the traveling-wave solution

$$
\phi=\frac{1}{2}\left[1-\tanh \left(\frac{z-V t}{2 \ell_{A}}\right)\right]
$$

for a specific velocity given by

$$
V=-M_{1} \epsilon \beta_{A}(T) \sqrt{2 W_{A}}
$$


This has the form $V=\tilde{\mu}_{A}\left(T_{A}-T\right)$, with a kinetic coefficient $\tilde{\mu}_{A}$ given by

$$
\tilde{\mu}_{A}=\frac{6 M_{1} L_{A} \ell_{A}}{T_{A}} .
$$

The solute diffusion coefficient $D$ has the form

$$
D=M_{2} \frac{R T}{v_{m}}
$$

A constant value for the solute mobility coefficient $M_{2}$ results in a solute diffusion coefficient that is constant throughout the system.

To treat unequal diffusivities in the liquid and solid phases [36], the solute mobility coefficient must be assumed to depend on $\phi$, leading to an expression for $D(\phi)$ that takes the appropriate limiting values in each phase. One choice is obtained by linear interpolation of the bulk diffusivity through the interface,

$$
D(\phi)=D_{S} r(\phi)+D_{L}[1-r(\phi)]
$$

where $r(\phi)$ is a suitable smooth function with $r(0)=0$ and $r(1)=1$; for example, $r(\phi)=\phi$. Another possibility is to linearly interpolate $\ln D$ through the interface, which is consistent with a linear interpolation of activation energies if Arrhenius expressions are assumed for the solid and liquid diffusivity. This produces the expression

$$
D(\phi)=D_{L}\left(\frac{D_{S}}{D_{L}}\right)^{r(\phi)}
$$

which we also examine in our numerical computations.

Our numerical and asymptotic results provide a verification that the solute trapping occurs at a characteristic velocity $V_{D} \sim D_{I} / \ell_{A}$, where the interfacial diffusivity $D_{I}$ is approximately given by the value of $D(\phi)$ at $\phi=1 / 2$. This suggests the consideration of a three-parameter model for the diffusivity in which $D(1 / 2)=D_{I}$ appears as a parameter independent of $D_{S}$ and $D_{L}$, as in

$$
D(\phi)=D_{S} r(\phi)+D_{L}[1-r(\phi)]+\left\{D_{I}-\left[D_{L}+D_{S}\right] r(1 / 2)\right\} s(\phi),
$$

where $s(\phi)$ is a smooth function that vanishes for $\phi=0$ and $\phi=1$ and is unity for $\phi=1 / 2$; an example is given by the basic double well potential $s(\phi)=16 g(\phi)$. We also include numerical results for $D(\phi)$ of this form.

\section{Numerical Solution}

We have numerically integrated the governing equations

$$
\frac{-V}{M_{1}} \frac{d \phi}{d z}=\epsilon^{2} \frac{d^{2} \phi}{d z^{2}}-\frac{\partial f}{\partial \phi}(\phi, c, T),
$$




$$
-V\left(c-c_{S}\right)=c(1-c) M_{2} \frac{d}{d z}\left[\frac{\partial f}{\partial c}(\phi, c, T)\right] .
$$

We used a finite difference discretization of the derivatives on a large finite interval with Neumann boundary conditions for $\phi$ and a mixed boundary condition for $c$ that admits the appropriate far field decay for the solute field. The resulting nonlinear equations are solved using Newton's method; details are given in Ref. [44]. For the purposes of illustrating the behavior of the phase-field model we used the material parameters given in Table 1 unless otherwise noted; these values are similar to those employed in WBM1, but with the same surface energy for each pure component. The far-field concentration was set to $c_{\infty}=0.0717441$, which corresponds to a solidus temperature of $1700 \mathrm{~K}$. For these parameters, the dilute limit of the ideal solution model yields an equilibrium partition coefficient of $k_{E}=0.7965$ and an equilibrium liquidus slope of $m_{L}=-310.9 \mathrm{~K}$.

\subsection{Results for $D(\phi)$ linear in $\phi$}

In Figure 1 we show representative computed concentration profiles for several values of the interface velocity $V$, holding the other parameters fixed. We have used the expression (50) for $D(\phi)$, with $D_{S} / D_{L}=10^{-5}$ and $r(\phi)=\phi$. In a sharp interface model with equilibrium interface conditions, as the velocity increases the maximum concentration in the liquid at the interface would be fixed at 0.09 , and the length scale $D_{L} / V$ of the solute profiles in the liquid would progressively shorten. For the phasefield model, Figure 1 shows that at low velocities the solute profile is similar to that given by the sharp interface model. However, as the interface velocity increases, not only does the solute decay length diminish, but the maximum value of the solute concentration decreases as well, indicating a reduction of the segregation of solute near the interfacial region and therefore the presence of solute trapping. We observe that solute trapping occurs when the interface velocity is large enough that $V \ell_{A} / D_{L}$ becomes of order unity. Since for this example the diffusion coefficient at the interface position $\phi=1 / 2$ is given by the arithmetic mean of $D_{L}$ and $D_{S}$, which is approximately $D_{L} / 2$ in this case, this result is consistent with the expectation that trapping will occur on a velocity scale $D_{I} / \ell_{A}$.

In contrast to the solute profiles shown in Figure 1 the corresponding phase-field profiles are almost identical over this range of velocities. This is because the velocity-dependent term on the left hand side of Eq. (53) is negligible for the range of interface velocities employed in Figure 1, because of the relatively large magnitude of $M_{1}$. Indeed, this is why it is reasonable to view $a$ in Eq. (35) as nearly constant. 
For our diffuse interface model with $k_{E}<1$ we adopt the definition

$$
k=\frac{c_{\infty}}{c_{\max }}
$$

for the nonequilibrium partition coefficient, where $c_{\max }$ is the maximum value of the concentration. (When $k_{E}>1$ an analogous definition is obtained by replacing $c_{\max }$ by the minimum concentration $c_{\text {min }}$ ) This definition reproduces the correct limiting behavior in the limit of small growth rates, in which case $c_{\infty}=c_{S}$ and $c_{\max }=c_{L}$ correspond to the appropriate equilibrium values for the solid and liquid concentration. It also exhibits the appropriate high-velocity limit $k \rightarrow 1$ as the concentration becomes uniform with $c_{\max }=c_{\infty}$. This definition for $k$ assumes that the maximum value of the concentration profile is the appropriate analogue to the liquid interfacial concentration in a sharp interface model. If significant interface adsorption were to occur, it may be difficult to separate this effect from the effect of bulk solute segregation at the moving interface. In that case this definition of the partition coefficient may be inadequate, and an alternative definition must be employed. Our assumption that $\sigma_{A}=\sigma_{B}$ is intended to circumvent such ambiguities by reducing the driving force for adsorption at the interface. We will return to this question below.

As can be seen in Figure 1, $k$ increases towards unity as the interface velocity increases. To quantify this dependence more directly, we have conducted a series of computations in which $k$ was computed in this way for various velocities. The results are shown as the data points in Figure 2. Also shown by the solid curve in Figure 2 is the expression given by the CG model,

$$
k=\frac{k_{E}+V / V_{D}}{1+V / V_{D}},
$$

where $V_{D} \ell_{A} / D_{L}=0.232$ is the normalized critical velocity predicted by a large-velocity asymptotic expansion described below. The CG model, coupled with the asymptotic expression for $V_{D}$, is seen to give an excellent description of the numerical results for solidification velocities ranging over six orders of magnitude. A least squares fit of the numerical data using the CG form with $V_{D}$ as a fitting parameter gives a value of $V_{D} \ell_{A} / D_{L}=0.244$, which is visually indistinguishable from the large-velocity result shown in Figure 2.

In Figure 3 the data points show the dependence of the computed interface temperature on the interface velocity. The three curves show the temperature predicted by the $\mathrm{CG}$ model as given in Eq. (17), with, from top to bottom, $\alpha=0$ (no solute drag), $\alpha=24 / 35$, and $\alpha=1$ (solute drag). The other parameters used in the CG model are the equilibrium values of $k_{E}=0.7965$ and $m_{L}=-310.9$ $\mathrm{K}$, and the value of $V_{D}=0.232 D_{L} / \ell_{A}$ determined from the asymptotic analysis. The intermediate 
value of $\alpha=24 / 35$ results from the asymptotic analysis of the large velocity limit described below, and is seen to give an excellent comparison with the numerical results for this case. ${ }^{2}$ At low velocities the temperature tends to the solidus temperature of $1700 \mathrm{~K}$. At intermediate velocities the temperature increases, tending toward the $T_{0}$ temperature of $1703 \mathrm{~K}$. Before the $T_{0}$ temperature is reached, the effect of interface kinetics becomes significant, and the temperature decreases rapidly for larger velocities.

\subsection{Results for $\log D(\phi)$ linear in $\phi$}

We next consider a diffusivity $D(\phi)$ given by expression (51) with $D_{S} / D_{L}=10^{-5}$ and $r(\phi)=\phi$. Computed concentration profiles for several values of the interface velocity $V$ are shown in Figure 4. In contrast to the results shown in Figure 1, the solute profiles in Figure 4 show that in this case trapping occurs at significantly lower values of the interface velocity; in fact, the trapping occurs before the characteristic length $D_{L} / V$ of the solute boundary layer has become comparable to the interface thickness. Since for this example the diffusion coefficient at the interface position $\phi=1 / 2$ is given by the geometric mean $\left(D_{L} D_{S}\right)^{1 / 2}$ of the liquid and solid diffusivities, this result remains consistent with the expectation that trapping will occur on a velocity scale $D_{I} / \ell_{A}$, which in this case is a much lower velocity due to the influence of $D_{S} \ll D_{L}$.

In Figure 5 the data points show the computed partition coefficients for this case. The solid curve in Figure 5 is the result of the CG model, where $V_{D} \ell_{A} / D_{L}=1.942 \times 10^{-2}$ is the normalized critical velocity predicted by the large-velocity asymptotic expansion described below. The CG model, coupled with the asymptotic expression for $V_{D}$, is found to give an adequate description of the numerical results for large solidification velocities, but there is significant qualitative disagreement for lower velocities. A least squares fit of the numerical data using the CG form with $V_{D}$ as a fitting parameter gives a value of $V_{D} \ell_{A} / D_{L}=4.467 \times 10^{-3}$, and is shown as the dashed curve in Figure 5 . The least squares value provides an overall improvement in the fit over the whole range of velocities, although the agreement is still rather unsatisfactory.

In Figure 6 we show the dependence of the interface temperature on the interface velocity and compare it to the temperature predicted by the CG model, both with and without solute drag. We have used the least square value $V_{D}=4.467 \times 10^{-3} D_{L} / \ell_{A}$ which gives better agreement than the asymptotic value for $V_{D}$, although here, as in Figure 5, the agreement is rather poor. The CG results do not appear to give good agreement with the numerical results for a diffusivity of the form (51).

\footnotetext{
${ }^{2}$ The asymptotic result for the temperature field assumes that $D_{S} / D_{L}=1$. The good agreement between this asymptotic result and the numerical data for $D_{S} / D_{L}=10^{-5}$ suggests insensitivity of the temperature to this ratio.
} 


\subsection{Results for $D_{I}$ independent of $D_{L}$ and $D_{S}$}

The results shown in Figures 1 and 4 suggest that the onset of trapping behavior occurs at characteristic velocities that scale with the ratio of the interface diffusivity $D_{I}$ to the interface width $\ell_{A}$. In the previous sections $D_{I}$ depends on $D_{L}$ and $D_{S}$. However, this is not necessarily so. In Figure 7 we show the effects of varying the interface diffusivity $D_{I}$ following the form given in Eq. (52) with fixed values of the bulk diffusivities, $D_{L}=D_{S}=10^{-5} \mathrm{~cm}^{2} \mathrm{~s}^{-1}$. The calculations are performed for a fixed velocity given by $V=0.86 D_{L} / \ell_{A}$, which is chosen so that significant trapping occurs for the ratio $D_{I} / D_{L}=1$ (dashed curve). The solute segregation across the interface is observed to be quite sensitive to the value of $D_{I}$; as $D_{I}$ increases, the characteristic trapping velocity $D_{I} / \ell_{A}$ becomes larger and the solute profile tends toward its equilibrium form. For $D_{I} / D_{L}<1$, the trapping effects become more pronounced as trapping is predicted to occur at lower speeds.

\subsection{Results for $\sigma_{A} \neq \sigma_{B}$}

The CG model predicts that the partition coefficient $k$ varies monotonically with $V$, which is consistent with the calculations shown in Figures 2 and 5 and available experimental data. However, Baker $[13,15]$ describes the possibility of non-monotonic dependence of the partition coefficient on velocity. He considers a model which assumes that the non-ideal part of the chemical potential, $\mu^{\prime}=\mu-$ $\left(R T / v_{m}\right) \ln [c /(1-c)]$, varies through the interfacial region in a prescribed manner. A non-monotonic dependence for $k(V)$ is predicted if $\mu^{\prime}(x)$ assumes a maximum or minimum in the interface region. This follows a suggestion by Chernov [5] that solute trapping might be associated with a state of low solute energy near the interface, which leads to a solute driving force in the liquid towards the interface, prior to incorporation of solute into the solid phase. In our model we have

$$
\mu^{\prime}=3\left\{\frac{\sigma_{B}}{\ell_{B}}-\frac{\sigma_{A}}{\ell_{A}}\right\} g(\phi)+\left\{\frac{L_{B}\left(T-T_{B}\right)}{T_{B}}-\frac{L_{A}\left(T-T_{A}\right)}{T_{A}}\right\} p(\phi),
$$

and nonmonotonic behavior of $\mu^{\prime}$ occurs if the contribution from the first term, proportional to the nonmonotonic function $g(\phi)$, is large enough. In the calculations discussed so far, we have taken $\sigma_{A}=\sigma_{B}$ and $\ell_{A}=\ell_{B}$ [and so $W_{A}=W_{B}$ by Eq. (43)]. The first term is then absent, resulting in a monotonic profile for $\mu^{\prime}$. We now consider the effects of varying the ratio $\sigma_{A} / \sigma_{B}$. To avoid complications with our definition of the segregation coefficient $k=c_{\infty} / c_{\max }$ when $c_{\max }$ is affected by positive interface adsorption effects (see discussion), which would be expected to occur for $\sigma_{B} / \sigma_{A}<1$, we consider instead the opposite case with $\sigma_{B} / \sigma_{A}>1$. 
In Figure 8 we show the partition coefficient $k=c_{\infty} / c_{\max }$ versus the normalized interface velocity $V \ell_{A} / D_{L}$ obtained from our numerical computations for various ratios of the surface tensions $\sigma_{B} / \sigma_{A} \geq$ 1. The calculations are performed with $D(\phi)$ constant to simplify the interpretation of the results by eliminating the competing effects of diffusivity variation across the interface. As $\sigma_{B} / \sigma_{A}$ increases from unity, the equilibrium solute profile corresponding to a stationary interface develops a minimum in concentration in the interfacial region, since this produces a lower surface energy while still retaining the equilibrium concentrations of the bulk phases. This minimum persists under finite rates of solidification, and the non-equilibrium solute profiles then exhibit both a maximum (due to solute rejection) and a minimum (due to negative interface adsorption) in concentration near the interfacial region. For a fixed velocity, as the ratio $\sigma_{B} / \sigma_{A}$ increases, the maximum concentration increases, and becomes large enough that the associated partition coefficient can be less than the equilibrium value, $k<k_{E}$, as shown in the figure for $\sigma_{B} / \sigma_{A}=4$.

\section{Large $V$ asymptotics}

In order to help interpret the numerical results presented above in which solute trapping is significant, we now describe results of an asymptotic expansion of the solution in the large velocity limit. We focus on the range of velocities for which trapping effects are significant and the effects of attachment kinetics are small; details are given in the Appendix. Here we summarize the results in the dilute solution limit $c_{\infty} \ll 1$. The phase-field solution is given to leading order by

$$
\phi(z)=\phi^{(0)}(z)+O(1 / V)
$$

where $\phi^{(0)}$ is the planar solution,

$$
\phi^{(0)}(z)=\frac{1}{2}\left[1-\tanh \left(\frac{z}{2 \ell_{A}}\right)\right] .
$$

The concentration is then given by

$$
c(z)=c_{\infty}+\frac{3}{8} c_{\infty}\left[\frac{D\left(\phi^{(0)}(z)\right)}{V \ell_{A}}\right] \ln \left(1 / k_{E}\right) \operatorname{sech}^{4}\left(\frac{z}{2 \ell_{A}}\right)+O\left(1 / V^{2}\right),
$$

and the temperature is given by

$$
T=T_{0}-V / \tilde{\mu}_{A}+\frac{9}{35} m_{L} c_{\infty}\left[\frac{D_{I}}{V \ell_{A}}\right] \frac{\ln ^{2}\left(1 / k_{E}\right)}{\left(1-k_{E}\right)}+O\left(1 / V^{2}\right),
$$

where the $T_{0}$ temperature is given by

$$
T_{0}=T_{A}+m_{L} c_{\infty} \frac{\ln \left(1 / k_{E}\right)}{\left(1-k_{E}\right)} .
$$


In deriving the expression for the temperature field we have assumed that the diffusivity is constant and denoted by $D_{I}\left(D_{I}=D_{L}=D_{S}\right)$; for a general diffusivity $D(\phi)$, the $O(1 / V)$ temperature correction is given by a solvability condition that is difficult to evaluate in closed form.

\subsection{Solute Trapping}

From the definition (55) of the partition coefficient for $k_{E}<1$, we find that in the large velocity limit $k$ may be represented as

$$
k=1-\frac{3}{8}\left[\frac{D\left(\phi^{(0)}\left(z_{m}\right)\right)}{V \ell_{A}}\right] \ln \left(1 / k_{E}\right) \operatorname{sech}^{4}\left(\frac{z_{m}}{2 \ell_{A}}\right)+O\left(1 / V^{2}\right),
$$

where we have used the expression (60) to evaluate the maximum value of the solute concentration, which is assumed to occur at $z=z_{m}$, with $c\left(z_{m}\right)=c_{\max }$. The appropriate value for $z_{m}$ depends on the specific form that is assumed for $D(\phi)$. If the diffusivity is constant, then the maximum occurs at $z_{m}=0$, leading to the expression

$$
k=1-\frac{3}{8}\left[\frac{D_{I}}{V \ell_{A}}\right] \ln \left(1 / k_{E}\right)+O\left(1 / V^{2}\right)
$$

where $D_{I}$ denotes the constant value of the diffusivity. ${ }^{3}$

The Aziz trapping function for $k_{E}<1$,

$$
k=\frac{k_{E}+V / V_{D}}{1+V / V_{D}}
$$

can be approximated by

$$
k \approx 1-\left(1-k_{E}\right)\left[\frac{V_{D}}{V}\right]+O\left(\left[\frac{V_{D}}{V}\right]^{2}\right)
$$

for $V_{D} / V \ll 1$. Comparing Eq. (64) and Eq. (66) gives that

$$
V_{D}=\frac{3}{8}\left[\frac{D_{I}}{\ell_{A}}\right] \frac{\ln \left(1 / k_{E}\right)}{\left(1-k_{E}\right)} .
$$

Comparing the large velocity expansions for the phase-field model and the Aziz trapping function thus gives a prediction that the trapping velocity $V_{D}$ depends not only on the interface diffusivity and thickness but also upon the equilibrium partition coefficient, a trend noted experimentally by Smith \& Aziz [45].

\footnotetext{
${ }^{3}$ This expression is also appropriate if the assumed form of a non-constant diffusivity $D(\phi)$ still gives rise to a maximum in $c(z)$ at $z_{m}=0$; in that case, $D_{I}=D(1 / 2)$ denotes the value of the diffusivity at $\phi=1 / 2$. For example, this expression applies for the case (50) with a function $r(\phi)$ that has $r^{\prime}(1 / 2)=0$, which makes $d c / d z$ vanish at $z=0$. This expression also applies to the case (52) with $D_{I}=D(1 / 2)$; in this case, the resulting expression is independent of $D_{L}$ and $D_{S}$.
} 
For the choice $r(\phi)=\phi$ in Eq. (50) for $D(\phi)$, the solute maximum generally occurs for $z_{m} \neq 0$, and the resulting expression for $V_{D}$ is more complicated. The result may be expressed in the form

$$
V_{D}=\frac{3}{8}\left[1-\left(t^{\star}\right)^{2}\right]^{2}\left[\frac{D_{L}\left[\left(1+t^{\star}\right) / 2\right]+D_{S}\left[\left(1-t^{\star}\right) / 2\right]}{\ell_{A}}\right] \frac{\ln \left(1 / k_{E}\right)}{\left(1-k_{E}\right)}
$$

where

$$
t^{\star}=\frac{2}{5} \frac{1+\Delta}{1-\Delta}\left(-1+\sqrt{1+\frac{5}{4}\left(\frac{1-\Delta}{1+\Delta}\right)^{2}}\right)
$$

is the value of $\tanh \left(z / 2 \ell_{A}\right)$ for $z=z_{m}$, and $\Delta=D_{S} / D_{L}$. Expression (68) has the same general form as expression (67), with $D_{I}$ given by a weighted arithmetic mean of $D_{S}$ and $D_{L}$. For the limiting case when the solute diffusivity of both phases are equal, $\Delta \rightarrow 1$, we find that $t^{\star} \rightarrow 0$ and $z_{m} \rightarrow 0$, and the expression (68) reduces to (67). In the more realistic case when the solid diffusivity is much less that liquid diffusivity, $\Delta \ll 1$, the interface diffusivity may be approximated by setting $\Delta=0$ in which case $V_{D}$ assumes the form

$$
V_{D}=\frac{648}{3125} \frac{D_{L} \ln \left(1 / k_{E}\right)}{\ell_{A}\left(1-k_{E}\right)} \approx 0.207 \frac{D_{L} \ln \left(1 / k_{E}\right)}{\ell_{A}\left(1-k_{E}\right)} .
$$

The expressions (68) and (70) both produce the value $V_{D} \ell_{A} / D_{L}=0.232$ that is used in Figure 2 to compare the numerical computations with the Aziz trapping function.

For the choice $r(\phi)=\phi$ in Eq. (51) for $D(\phi)$, the extremum may be found from Eq. (60), resulting in the expression

$$
V_{D}=\frac{3}{8}\left[1-\left(t^{\star}\right)^{2}\right]^{2}\left[\frac{\sqrt{D_{L}^{\left(1+t^{\star}\right)} D_{S}^{\left(1-t^{\star}\right)}}}{\ell_{A}}\right] \frac{\ln \left(1 / k_{E}\right)}{\left(1-k_{E}\right)}
$$

where now

$$
t^{\star}=\frac{4}{\ln \Delta}+\sqrt{1+\left(\frac{4}{\ln \Delta}\right)^{2}} \text {. }
$$

Expression (71) has the same general form as expression (67), with $D_{I}$ given by a weighted geometric mean of $D_{S}$ and $D_{L}$. This expression is used to produce the value $V_{D} \ell_{A} / D_{L}=1.942 \times 10^{-2}$ that is used in Figure 5 to compare the numerical computations with the Aziz trapping function for this case.

The two expressions for $V_{D}$ both involve weighted means of $D_{S}$ and $D_{L}$, which are determined by the specific form of $D(\phi)$ that is assumed through the interface region. This need not be the case; in particular, the use of a more complicated model for $D(\phi)$ such as given by Eq. (52) would produce an expression for $V_{D}$ that is independent of $D_{S}$ and $D_{L}$.

The expression for $V_{D}$ given by Eq. (67) holds for $k_{E}<1$. A similar analysis may be conducted for for $k_{E} \geq 1$, in which case we find that

$$
V_{D}=\frac{3}{8} \frac{D_{I} \ln \left(k_{E}\right)}{\ell_{A}\left(1-1 / k_{E}\right)}, \quad\left(k_{E} \geq 1\right)
$$


Thus $V_{D}$ is predicted to increase as $k_{E}$ deviates from unity in either direction.

Finally, in Figure 9 we compare the experimental data for $V_{D}$ obtained by Aziz and coworkers (see [58]) for both silicon and aluminum alloys to the quantity $\ln k_{E} /\left(k_{E}-1\right)$. The correlation indicates that our theory is in qualitative agreement with the experimental results, correctly predicting an increase in $V_{D}$ with decreasing equilibrium partition coefficient. The considerable scatter apparent in the plot may be due to the unknown values of $D_{I}$.

\subsection{Solute Drag}

Here we limit our attention to the case $k_{E}<1$ and $D_{S}=D_{L}$. The interface temperature in the dilute limit given by Eq. (61) may be expressed as

$$
T=T_{0}-V / \tilde{\mu}_{A}+\frac{24}{35} \frac{V_{D}}{V} m_{L} c_{\infty} \ln \left(1 / k_{E}\right)+\ldots
$$

where we have used the expression for $V_{D}$ in Eq. (67). The temperature in Eq. (17) that is predicted by the CG model may be expanded for large $V / V_{D}$ to yield that

$$
T=T_{0}-V / \tilde{\mu}_{A}+\alpha \frac{V_{D}}{V} m_{L} c_{\infty} \ln \left(1 / k_{E}\right)+\ldots
$$

in the dilute limit. Comparing these two expressions for the interface temperature we find that $\alpha=$ 24/35, the value used in Figs. 3 and 6 to compare the numerical computations with the predictions of the Aziz CG model. This nonzero value for $\alpha$ confirms that solute drag is present in the phase-field model, as is consistent with the analysis of Section 2.2.2. In general, we would expect the specific value obtained for $\alpha$ to depend on the choice for $D(\phi)$.

\section{Discussion}

The formulation of a phase transformation using a single free energy function and self consistent postulates about the kinetics has distinct advantages. Among them the controversy regarding the necessity to include or exclude solute drag is resolved quite naturally. Early models that treated solute drag (e.g. Cahn [17]) were applied to grain growth in impure solids. Here a separate driving force for

grain growth was assumed to exist (e.g., induced by curvature differences) and one sought to determine how much of that driving force remained available for the motion of the grain boundary. Calculation of the solute diffusion process and the dissipation of energy were thus performed to determine the reduction of the driving force. In the solidification literature a similar postulate has been made about 
the existence of a separate driving force for solidification [Eq. (5)]. Hence the solute drag is normally computed separately and used to erode the driving energy. The results of Eqs. (37) and (38) show the natural reduction of the driving force by the dissipation.

We have investigated the effect of different assumptions regarding the variation of the diffusion mobility through the interfacial region by varying the form of the function $D(\phi)$. These differences change the velocity at which solute trapping becomes important, and also presumably changes the amount of dissipation in the interfacial region due to the diffusion processes. Indeed we determined that a linear interpolation for $\mathrm{D}$ between the liquid value and the solid value across the interface produces predictions quite similar to those of Aziz, a model which has been subject to significant experimental validation.

We have not investigated the sensitivity of the results to the form of the double well to any significant degree. We have, however, shown that changing the relative heights of the double-well potential (by changing $\sigma_{A}$ and $\sigma_{B}$ ) of the two pure components can lead to a nonmonotonic $k(V)$ function. In general the use of different double well potentials in (40) as well as a non-ideal solution model would likely alter the quantitative predictions of our analysis. The form (40) that we have chosen for the free energy function allows the one-dimensional traveling wave solution (46) for all velocities [59]. Replacing the cubic function $p(\phi)=\phi^{2}(3-2 \phi)$ used in $(40)$ by the quintic function $p(\phi)=\phi^{3}\left(10-15 \phi+6 \phi^{2}\right)$ as in the treatment of Wang et al. [60] relieves the restrictions $|\beta(T)|<1 / 2$, but also produces a onedimensional traveling wave whose shape is no longer independent of velocity; this may require changes in the interpretation of $\tilde{\mu}$ and $V_{D}$.

In addition to the more usual case where $k_{E}<1$, we briefly investigated the case where $k_{E}>1$. The appropriate form (12) of the Aziz formulation for this case was compared with the present model. It was established that $V_{D}$ should be smallest for alloys with $k_{E}$ near unity and increase for alloys with $k_{E}$ much less than or much greater than unity. A functional form was predicted for the $k_{E}$ dependence of $V_{D}$ which seems to correlate well with experiments (Fig. 9).

We have some concerns about our definition of the nonequilibrium partition coefficient as we extract information about the prediction of the phase-field model. For $k_{E}<1$, our definition of $k(V)$ is based on identifying the liquid concentration at the interface, $c_{L}$, with the solute maximum $c_{\max }$ of the entire solute profile. Alternate definitions were tried, such as equating the total amount of solute above $c_{\infty}$ in the interfacial region and in the bulk liquid to the total solute above $c_{\infty}$ for an exponential profile at a sharp interface freezing at the same speed. This was found to be unsatisfactory because this alternate definition does not reproduce the correct limit $k \rightarrow 1$ for large velocities, as can be seen from the 
form of the asymptotic expansion for $c(z)$ in our model. The concern about our definition was clearly manifest when we examined situations where significant interface adsorption is present. For example, for a stationary interface at equilibrium, positive surface adsorption leads to a value of $c_{\max }$ larger than the equilibrium value of $c_{L}$ present in the bulk of the liquid, so that the definition (55) leads to an inappropriate value for $k$ in this simple case. We therefore confined our attention to cases of negative interface adsorption with $k_{E}<1$, where the identification of $c_{L}$ with $c_{\max }$ at least does not lead to trivial inconsistencies.

We have also performed melting simulations to examine whether very small values of the solid diffusivity $D_{S}$ lead to significantly smaller values of $V_{D}$. The results are identical to those observed for the solidification case, in that the simulation results for $V_{D}$ are found to depend on the values of $D(\phi)$ in the interfacial region and not necessarily on the bulk values of $D_{L}$ and $D_{S}$. This is also clear from the asymptotic analysis results: the large velocity analysis holds for either solidification or melting, and similar conclusions are obtained for both cases. For example, with the linear form (50) for $D(\phi)$, computed values for $V_{D}$ are found to scale with the arithmetic mean $\left(D_{L}+D_{S}\right) / 2$ of the bulk diffusivities, and with $D_{S} \ll D_{L}$ the numerical values of $V_{D}$ for both solidification and melting are roughly $D_{L} / 2$. In general, the predicted values for $V_{D}$ are sensitive to the assumed form for $D(\phi)$.

Aziz and coworkers (see [58]) have observed that the experimental values of $V_{D}$ (for solidification) do not correlate strongly with either $D_{L}$ or $D_{S}$. These findings are consistent with our results for models of $D(\phi)$ in which $D_{I}$ is uncorrelated to $D_{L}$ or $D_{S}$.

\section{Conclusions}

1. Solutions to phase-field governing equations for alloy solidification with a finite interface thickness that neglect the gradient energy due to concentration exhibit solute trapping and dissipation due to solute drag.

2. In particular, it is shown that

(a) the governing equation for the concentration recovers the sharp interface notion that the jump in interdiffusion potential across the interface depends on the velocity, leading to the velocity dependence of the partition coefficient,

and

(b) the governing equation for the phase field recovers the notion that the velocity 
depends on the driving force for solidification following the tangent-to-curve rule with dissipation due to solute drag, leading to the velocity dependence of the interface temperature.

3. Numerical results as well as high velocity asymptotic results for the velocity dependence of temperature, $T(V)$, and solute partitioning, $k(V)$, were explored for a particular choice of double-well potential, an ideal solution, linear dependence of the diffusion coefficient on the phase field and similar barrier heights for the pure components; i.e., $W_{A}$ and $W_{B}$. Under these conditions the results agree closely with the CG model of Aziz and Kaplan for a particular choice of their values for:

(a) the critical speed for trapping, $V_{D}$, that depends on $k_{E}$; viz., proportional to $\ln k_{E} /\left(k_{E}-1\right)$ for $k_{E}<1$ and approximately proportional to the arithmetic mean of the liquid and solid diffusion coefficients. Such a trend for the dependence of $V_{D}$ on $k_{E}$ has been seen experimentally by Smith \& Aziz.

(b) the amount of dissipation due to solute drag, $\alpha$, of approximately $24 / 35$.

Numerical calculations performed with a linear dependence of the logarithm of the diffusion coefficient on the phase field did not agree with the CG model of Aziz and Kaplan.

4. Depending on the choice of double well potential; viz., the barrier heights of the two components, more complex behavior of the effective $k(V)$ relation is predicted by the phase-field model, including nonmonotonic behavior similar to that exhibited by the Baker-Cahn and Hillert-Sundman models.

\section{Appendix}

In the limit of high solidification rates it is possible to obtain approximate expressions for the interface temperature and solute profiles. In order to understand the numerical results presented above in which solute trapping is significant but the effects of kinetics are comparatively minor, we consider an asymptotic solution in the limit of large velocities where $V$ is identified as being of a magnitude so that $D_{L} / \ell_{A} \ll V \ll \tilde{\mu}_{A} T_{0}$. Hence we consider the simplified set of equations in which the effects of interface attachment kinetics are eliminated by setting $1 / M_{1}=0$. This removes the term $V / \tilde{\mu}_{A}$ from the leading order expression for the interface temperature, and simplifies the subsequent analysis. For simplicity 
we also consider the case $\sigma_{A}=\sigma_{B}$; the more general analysis for the case $\sigma_{A} \neq \sigma_{B}$ is given in [44]. We find that the resulting large velocity analysis with $1 / M_{1}=0$ is a useful limit for understanding the solute trapping that is observed experimentally in metals for velocities on the order of meters per second.

We perform an asymptotic expansion for large velocities by expanding the variables in the form

$$
\begin{aligned}
\phi & =\phi^{(0)}+\frac{1}{V} \phi^{(1)}+\ldots, \\
c & =c^{(0)}+\frac{1}{V} c^{(1)}+\ldots, \\
T & =T^{(0)}+\frac{1}{V} T^{(1)}+\ldots
\end{aligned}
$$

substituting these expansions into the governing equations, and solving the resulting equations order by order. The procedure is summarized in dimensional form, although the actual expansion is best performed in dimensionless variables.

At leading order the solute equation gives that $c^{(0)}=c_{\infty}$. The leading-order phase-field equation then becomes

$$
\epsilon^{2} \frac{d^{2} \phi^{(0)}}{d z^{2}}=f_{\phi}\left(\phi^{(0)}, c_{\infty}, T^{(0)}\right)
$$

which admits the planar solution

$$
\phi^{(0)}=\frac{1}{2}\left[1-\tanh \left(\frac{z}{2 \ell_{A}}\right)\right]
$$

where $\ell_{A}$ is the interface width given in Eq. (42), and where

$$
T^{(0)}=T_{0}=\frac{c_{\infty} L_{B}+\left(1-c_{\infty}\right) L_{A}}{\left[c_{\infty} L_{B} / T_{B}+\left(1-c_{\infty}\right) L_{A} / T_{A}\right]}
$$

is the temperature at which the bulk free energies of the liquid and solid phases are equal.

At first order the solute equation gives that

$$
c^{(1)}=\frac{-c_{\infty}\left(1-c_{\infty}\right)}{R T_{0} / v_{m}} D\left(\phi^{(0)}\right) \frac{d}{d \tilde{z}}\left[f_{c}\left(\phi^{(0)}, c_{\infty}, T_{0}\right)\right]
$$

from which we find that

$$
c^{(1)}=\frac{3}{8}\left[\frac{L_{B}\left(T_{0}-T_{B}\right)}{T_{B}}-\frac{L_{A}\left(T_{0}-T_{A}\right)}{T_{A}}\right] \frac{c_{\infty}\left(1-c_{\infty}\right) D\left(\phi^{(0)}\right)}{\ell_{A} R T_{0} / v_{m}} \operatorname{sech}^{4}\left(\frac{z}{2 \ell_{A}}\right) .
$$

The first order phase-field equation has the form

$$
\epsilon^{2} \frac{d^{2} \phi^{(1)}}{d z^{2}}-f_{\phi \phi}\left(\phi^{(0)}, c_{\infty}, T_{0}\right) \phi^{(1)}=f_{\phi c}\left(\phi^{(0)}, c_{\infty}, T_{0}\right) c^{(1)}+f_{\phi T}\left(\phi^{(0)}, c_{\infty}, T_{0}\right) T^{(1)}
$$


which has a solvability condition

$$
0=\int_{-\infty}^{\infty} f_{\phi c}\left(\phi^{(0)}, c_{\infty}, T_{0}\right) c^{(1)} \phi_{z}^{(0)} d z+T^{(1)} \int_{-\infty}^{\infty} f_{\phi T}\left(\phi^{(0)}, c_{\infty}, T_{0}\right) \phi_{z}^{(0)} d z
$$

that allows the determination of $T^{(1)}$. We have

$$
\int_{-\infty}^{\infty} f_{\phi T}\left(\phi^{(0)}, c_{\infty}, T_{0}\right) \phi_{z}^{(0)} d z=-\left[c_{\infty} \frac{L_{B}}{T_{B}}+\left(1-c_{\infty}\right) \frac{L_{B}}{T_{B}}\right]
$$

and

$$
\begin{gathered}
\int_{-\infty}^{\infty} f_{\phi c}\left(\phi^{(0)}, c_{\infty}, T_{0}\right) c^{(1)} \phi_{z}^{(0)} d z=\int_{-\infty}^{\infty} c^{(1)} \frac{d}{d z}\left[f_{c}\left(\phi^{(0)}, c_{\infty}, T_{0}\right)\right] d z \\
=\frac{-R T_{0} / v_{m}}{c_{\infty}\left(1-c_{\infty}\right)} \int_{-\infty}^{\infty}\left[c^{(1)}(z)\right]^{2} \frac{d z}{D\left(\phi^{(0)}\right)} .
\end{gathered}
$$

The latter integral is difficult to evaluate in closed form for general diffusivities $D\left(\phi^{(0)}\right)$; for the case of constant diffusivities $D\left(\phi^{(0)}\right)=D_{I}$, however, the integral can be evaluated to yield the following expression for the first order temperature correction,

$$
T^{(1)}=\frac{-9}{35} \frac{c_{\infty}\left(1-c_{\infty}\right) D_{I} / \ell_{A}}{\left(R T_{0} / v_{m}\right)\left[c_{\infty} L_{B} / T_{B}+\left(1-c_{\infty}\right) L_{A} / T_{A}\right]}\left[\frac{L_{B}\left(T_{0}-T_{B}\right)}{T_{B}}-\frac{L_{A}\left(T_{0}-T_{A}\right)}{T_{A}}\right]^{2}
$$

To obtain the limiting forms of these expressions that are given in Section 5 , we make use of the results

$$
\ln \left(1 / k_{E}\right)=\frac{L_{B}\left(T_{A}-T_{B}\right)}{R T_{A} T_{B} / v_{m}}, \quad m_{L}=\frac{R T_{A}^{2}\left(k_{E}-1\right)}{v_{m} L_{A}}, \quad T_{0}=T_{A}+\frac{m_{L} c_{\infty} \ln \left(1 / k_{E}\right)}{1-k_{E}},
$$

that hold in the dilute solution limit.

\section{References}

[*] Present address: School Of Mathematical Sciences, Universiti Sains Malaysia, 11800 Minden, Penang, Malaysia.

[1] M. J. Aziz, J. Appl. Phys. 53, 1158 (1982).

[2] M. J. Aziz, Appl. Phys. Lett. 43, 552 (1983).

[3] M. J. Aziz and T. Kaplan, Acta Metall. 36, 2335 (1988).

[4] R. N. Hall, J. Phys. Chem. 57, 836 (1953).

[5] A. A. Chernov, in Growth of Crystals, Vol. 3 (edited by A. V. Shubnikov and N. N. Sheftal), p. 35. Consultants Bureau, New York (1962). 
[6] J. C. Brice, The Growth of Crystals from the Melt, p. 65. North Holland, Amsterdam (1965).

[7] R. F. Wood, Appl. Phys. Lett. 37, 302 (1980).

[8] K. A. Jackson, in Surface Modification and Alloying by Laser, Ion and Electron Beams, (edited by J. M. Poate, G. Foti and D. C. Jacobson), p. 51. Plenum Press, New York (1983).

[9] G. H. Gilmer, Mater. Res. Soc. Symp. Res. 13, 249 (1983).

[10] J. W. Cahn and S. M. Allen, J. Phys. (Paris) Colloque C7, C7-51 (1977).

[11] S. M. Allen and J. W. Cahn, Acta Metall. Mater. 27, 1085 (1979).

[12] J. W. Cahn and J. E. Hilliard, J. Chem. Phys. 28, 28 (1958).

[13] J. C. Baker, Interfacial Partitioning during Solidification, Ph. D. Thesis MIT (1970) Chapter V.

[14] J. C. Baker and J. W. Cahn, Thermodynamics of Solidification, in Solidification, ASM Seminar Series, (ASM, Metals Park, Ohio, 1971) pp. 23-58.

[15] J. W. Cahn, S. R. Coriell, and W. J. Boettinger, in Laser and Electron Beam Processing of Materials, (edited by C. W. White and P. S. Peercy) p. 89. Academic Press, New York (1980).

[16] M. Hillert and B. Sundman, Acta Metall. 24, 731 (1976).

[17] J. W. Cahn, Acta Metall. 10, 789 (1962).

[18] J. S. Langer, "Models of pattern formation in first-order phase transitions", in Directions in Condensed Matter Physics, edited by G. Grinstein and G. Mazenko, (World Scientific, Philadelphia, 1986), p. 165.

[19] G. Caginalp, in Applications of Field Theory to Statistical Mechanics, edited by L. Garrido, Lecture Notes in Physics Vol. 216 (Springer-Verlag, Berlin, 1985), p. 216.

[20] J. B. Collins and H. Levine, Phys. Rev. B 31, 6119 (1985).

[21] R. Kobayashi, Physica D 63, 410 (1993).

[22] R. Kobayashi, Experimental Math 3, 60 (1994).

[23] A. A. Wheeler, B. T. Murray and R. J. Schaefer, Physica D 66, 243 (1993). 
[24] A. Karma and W. J. Rappel, Phys. Rev. E 53, R3017 (1996).

[25] A. Karma and W. J. Rappel, Phys. Rev. Lett. 77, 4050 (1996).

[26] S-L. Wang and R. F. Sekerka, Phys. Rev. E 53, 3760 (1996).

[27] S-L. Wang and R. F. Sekerka, J. Comp. Phys. 127, 110 (1996).

[28] J. S. Langer and R. F. Sekerka, Acta Metall. 23, 1225 (1975).

[29] A. A. Wheeler, W. J. Boettinger, and G. B. McFadden, Phys. Rev. A 45, 7424 (1992).

[30] H. Löwen, J. Bechoefer, and L. S. Tuckerman, Phys. Rev. A 45, 2399 (1992).

[31] G. Caginalp and W. Xie, Phys. Rev. E. 48, 1897 (1993).

[32] T. Lin and R. C. Rogers, Comp. Mat. Sci. 4, 159 (1995).

[33] E. Fried and M. E. Gurtin, Physica D 68, 326 (1993).

[34] E. Fried and M. E. Gurtin, Physica D 72, 287 (1994).

[35] M. E. Gurtin, Arch. Rat. Mech. 131, 67 (1995).

[36] J. Warren and W. J. Boettinger, Acta Metall. Mater. 43, 689 (1995).

[37] A. A. Wheeler, G. B. McFadden and W. J. Boettinger, Proc. Roy. Soc. Lond. A 452, 495, (1996).

[38] A. Karma, Phys. Rev. E 49, 2245 (1994).

[39] K. R. Elder, Francois Drolet, J. M. Kosterlitz and Martin Grant, Phys. Rev. Lett. 72, 677 (1994).

[40] K. R. Elder, J. D. Gunton, and Martin Grant, Phys. Rev. E 54, 6476 (1996).

[41] I. Steinbach, F. Pezzolla, B. Nestler, M. Sesselberg, R. Prieler, G. J. Schmitz, and J.L.L. Rezenda, Physica D 94, 135 (1996).

[42] A. A. Wheeler, W. J. Boettinger, and G. B. McFadden, Phys. Rev. E. 47, 1893 (1993).

[43] A. A. Wheeler, N. A. Ahmad, W. J. Boettinger, R. J. Braun, G. B. McFadden, and B. T. Murray, Adv. Space Res. 16, 163 (1995).

[44] N. A. Ahmad, Phase-field model of rapid solidification of a binary alloy, Ph. D. Thesis, University of Southampton, UK (1997). 
[45] P. M. Smith and M. J. Aziz, Acta Metall. Mater. 42, 3515 (1994).

[46] M. Conti, Phys. Rev. E. 55, 701 (1997).

[47] M. Conti, Phys. Rev. E. 55, 765 (1997).

[48] P. C. Fife and C. Charach, preprint (1998).

[49] M. Plapp and J. F. Gouyet, Phys. Rev. E 55, 5321 (1997).

[50] M. Conti, preprint (1998).

[51] S. G. Kim, W. T. Kim, and T. Suzuki, preprint (1998).

[52] T. Kaplan, M. J. Aziz, and L. J. Gray, J. Chem. Phys. 90, 1133 (1989).

[53] W. J. Boettinger and M. J. Aziz, Acta Metall. 37, 3379 (1989).

[54] M. J. Aziz and W. J. Boettinger, Acta Metall. Mater. 42, 527 (1994).

[55] J. Ågren, Acta Metall. 37, 181 (1989).

[56] M. E. Gurtin and P. W. Voorhees, Acta Mater. 44, 235 (1996).

[57] Z.-K. Liu, in Solid-Solid Phase Transformations, W.C. Johnson, J. M. Howe, D.E. Laughlin, and W.A. Soffa (eds.) The Minerals, Metals, and Materials Soc. 1994 p. 219.

[58] M. J. Aziz, Metall. and Matls. Trans. 27A, 671 (1996).

[59] E. Montroll, in Statistical Mechanics, S. Rice, K. Freed, and J. Light (eds.) University of Chicago, Chicago, 1972.

[60] S.-L. Wang, R. F. Sekerka, A. A. Wheeler, B. T. Murray, S. R. Coriell, and G. B. McFadden, Physica D 69, 189 (1993). 
Table 1. Thermophysical properties used in calculations

\begin{tabular}{ll}
\hline$L_{A}$ & $2350 \mathrm{~J} \mathrm{~cm}^{-3}$ \\
$L_{B}$ & $1725 \mathrm{~J} \mathrm{~cm}^{-3}$ \\
$T_{A}$ & $1728 \mathrm{~K}$ \\
$T_{B}$ & $1358 \mathrm{~K}$ \\
$\sigma_{A}$ & $2.8 \times 10^{-5} \mathrm{~J} \mathrm{~cm}^{-2}$ \\
$\sigma_{B}$ & $2.8 \times 10^{-5} \mathrm{~J} \mathrm{~cm}^{-2}$ \\
$\tilde{\mu}_{A}$ & $242.8 \mathrm{~cm} \mathrm{~s}^{-1} \mathrm{~K}^{-1}$ \\
$D_{L}$ & $10^{-5} \mathrm{~cm}^{2} \mathrm{~s}^{-1}$ \\
$D_{S}$ & $10^{-10} \mathrm{~cm}^{2} \mathrm{~s}^{-1}$ \\
$\ell_{A}$ & $6.48 \times 10^{-8} \mathrm{~cm}$ \\
$\ell_{B}$ & $6.48 \times 10^{-8} \mathrm{~cm}$ \\
\hline
\end{tabular}




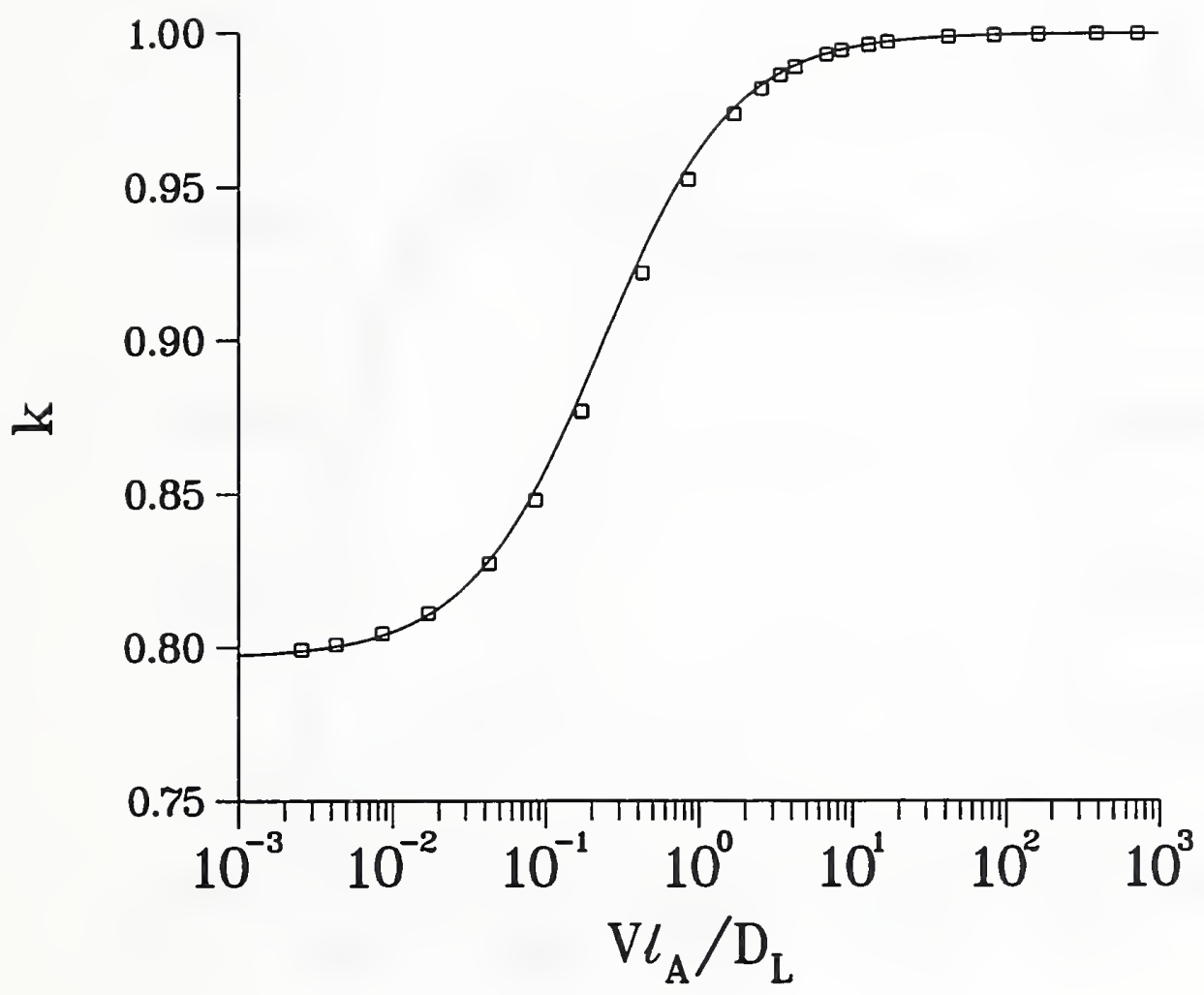

Figure 1: The open squares denotes the values of the partition coefficient $k=c_{\infty} / c_{\max }$ versus te normalized interface velocity $V \ell_{A} / D_{L}$ obtained from our numerical computations. The solid curve shows the corresponding dependence of $k$ on the interface velocity that is predicted by the CG model as given by Eq. (56) with $V_{D} \ell_{A} / D_{L}=0.232$ given by the large-velocity asymptotic limit in the expression Eq. (67). 


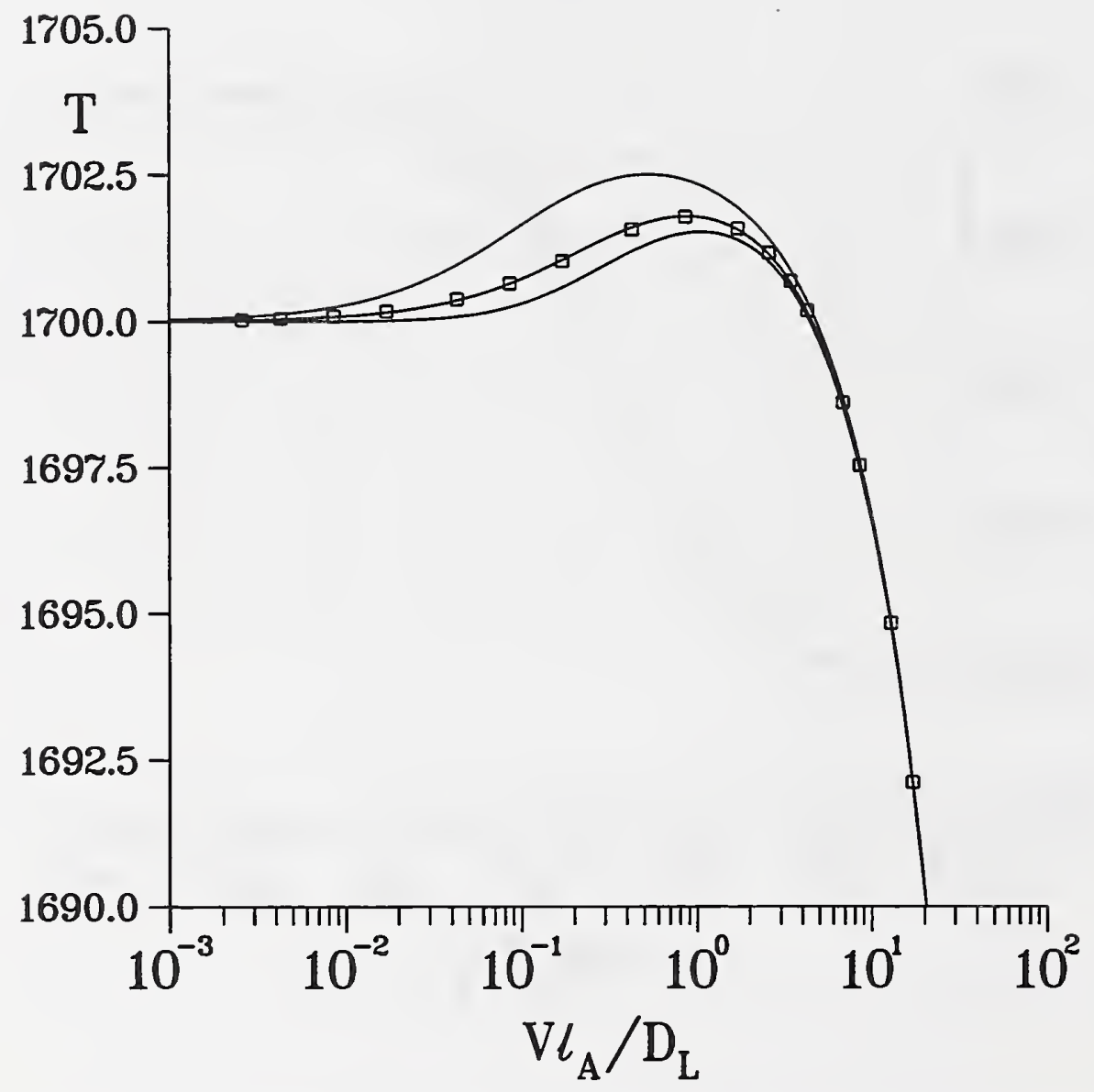

Figure 2: The data points denote the temperature obtained from our calculations for different values of growth velocity $V$. The upper curve shows the temperature given by Eq. (17) without solute drag $(\alpha=0)$, the lower curve shows the temperature given by this expression with solute drag $(\alpha=1)$, and the middle curve shows the temperature given by this expression with the asymptotic value $(\alpha=24 / 35)$. 


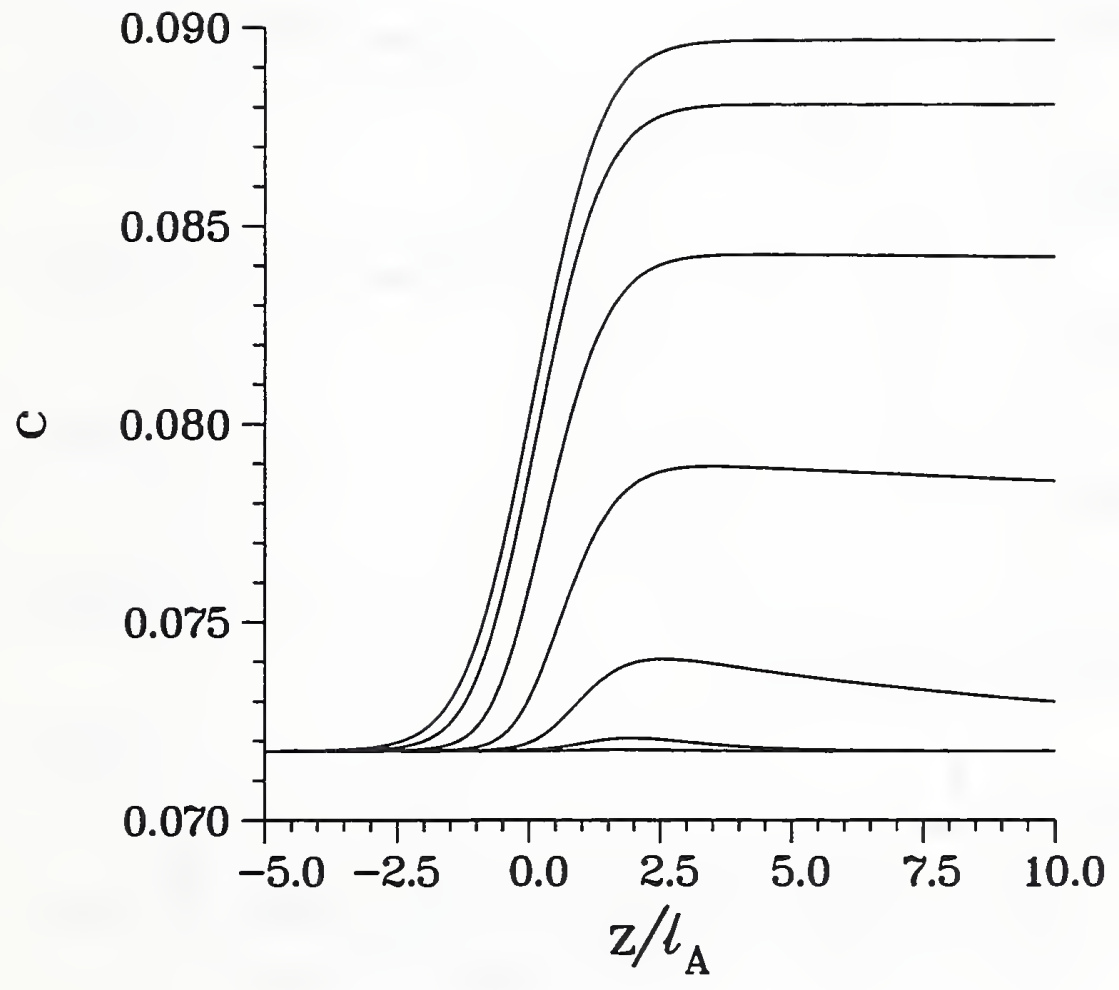

Figure 3: This figure shows the computed solute profiles for six different values of the interface velocity $V$ obtained by using the logarithmic model Eq. (51) for $D(\phi)$ with $D_{S} / D_{L}=10^{-5}$. The values of $V \ell_{A} / D_{L}$ are $8.58 \times\left(10^{-3}\right), 8.58 \times\left(10^{-2}\right), 0.429,0.859,2.58$ and 8.58 . The curves are ordered so that the interface velocity $V$ increases from the top to the bottom curve. 


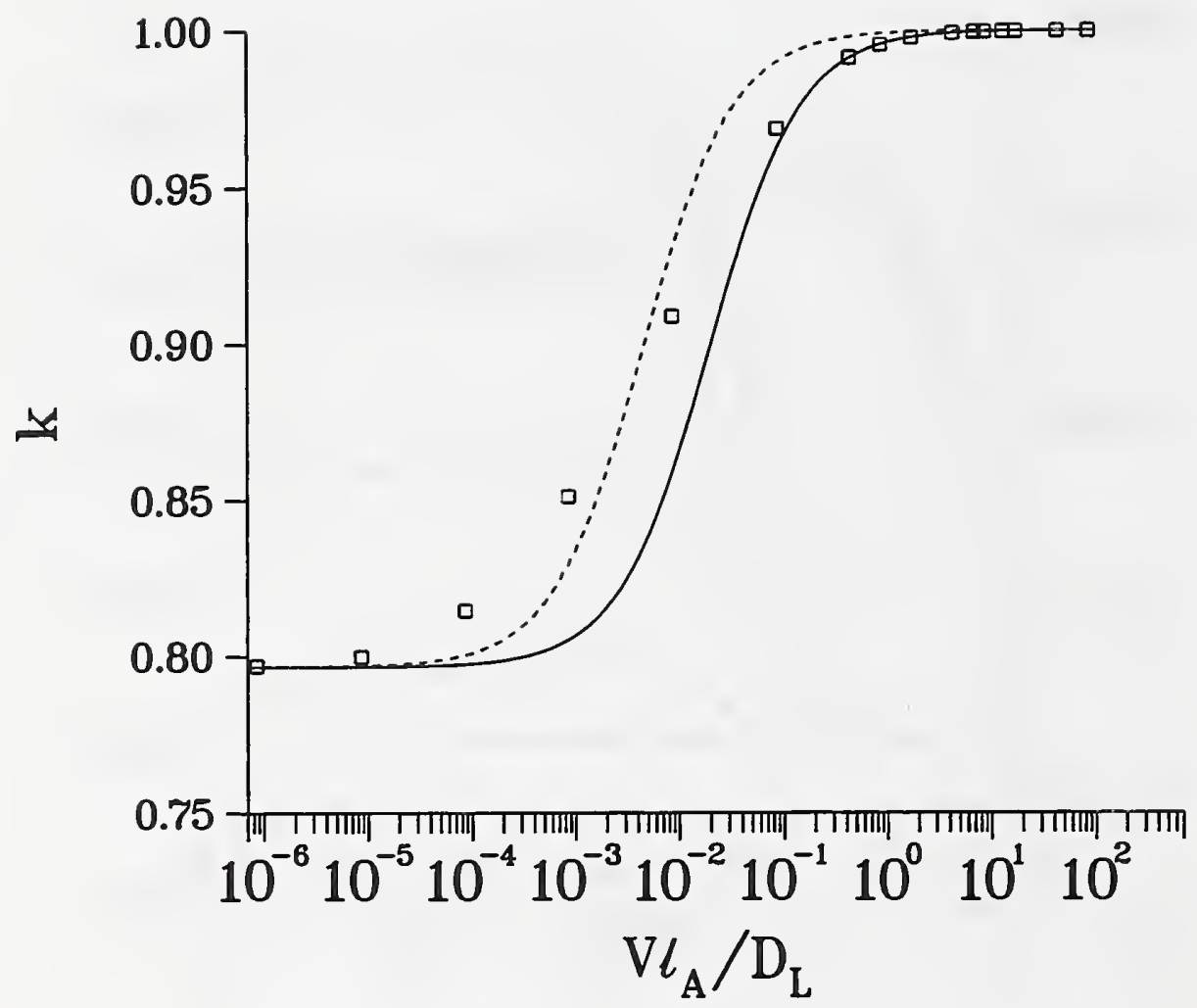

Figure 4: The open squares denotes the values of the partition coefficient $k=c_{\infty} / c_{\max }$ versus the normalized interface velocity $V \ell_{A} / D_{L}$ obtained from our numerical computations by using the logarithmic model Eq. (51) for $D(\phi)$ with $D_{S} / D_{L}=10^{-5}$. The solid curve shows the corresponding dependence of $k$ on the interface velocity that is predicted by the CG model as given by Eq. (56) with $V_{D} \ell_{A} / D_{L}=1.942 \times 10^{-2}$ given by the large-velocity asymptotic limit in the expression Eq. (67). The dashed curved represents the same CG form but with a least square fit value $V_{D} \ell_{A} / D_{L}=4.467 \times 10^{-3}$. 


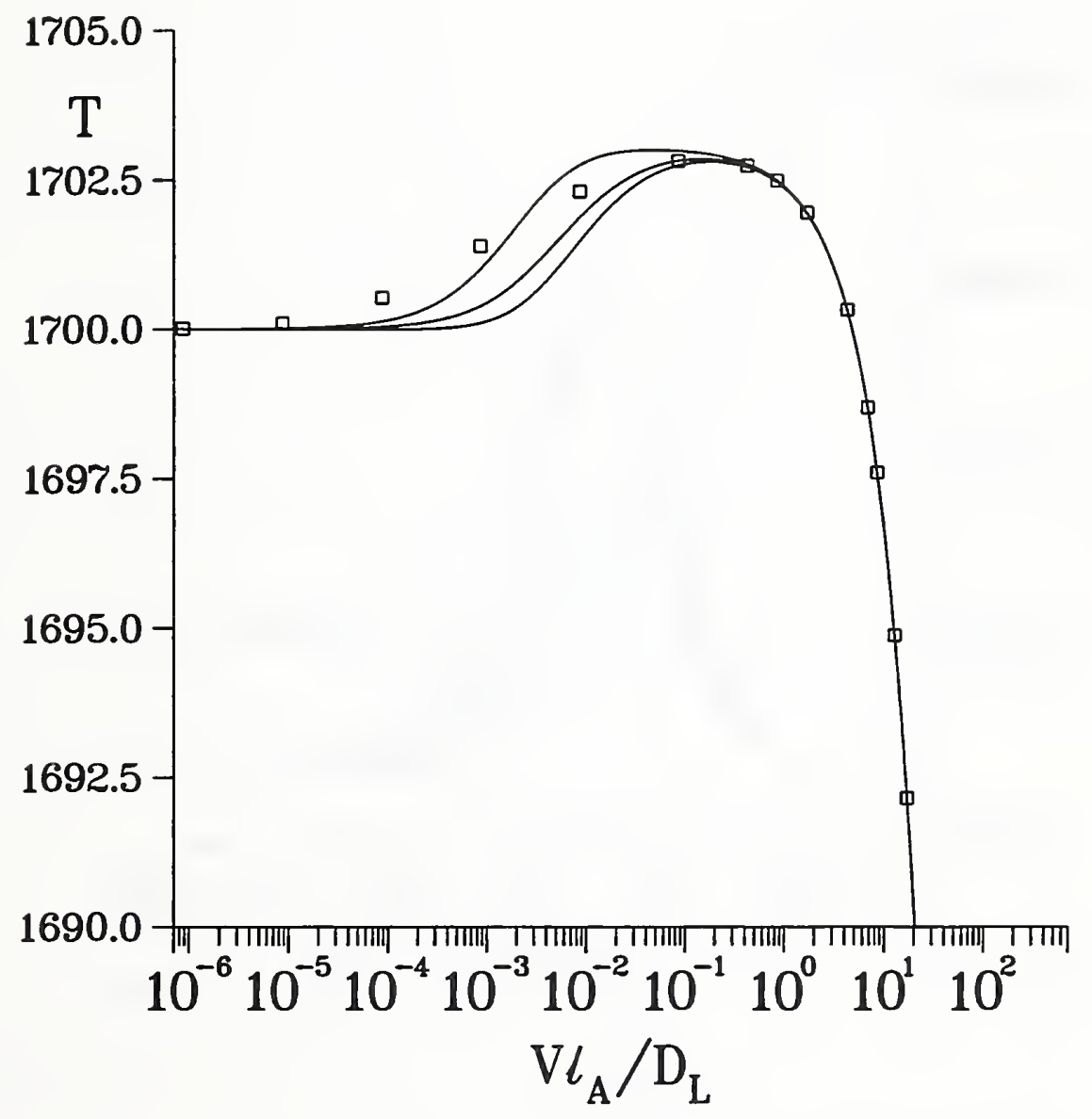

Figure 5: The data points denote the temperature obtained from our calculations for different values of growth velocity $V$ by using the logarithmic model Eq. (51) for $D(\phi)$ with $D_{S} / D_{L}=10^{-5}$. The upper curve shows the temperature given by Eq. (17) without solute drag $(\alpha=0)$, the lower curve shows the temperature given by this expression with solute drag $(\alpha=1)$, and the middle curve shows the temperature given by this expression with the asymptotic value $(\alpha=24 / 35)$. 


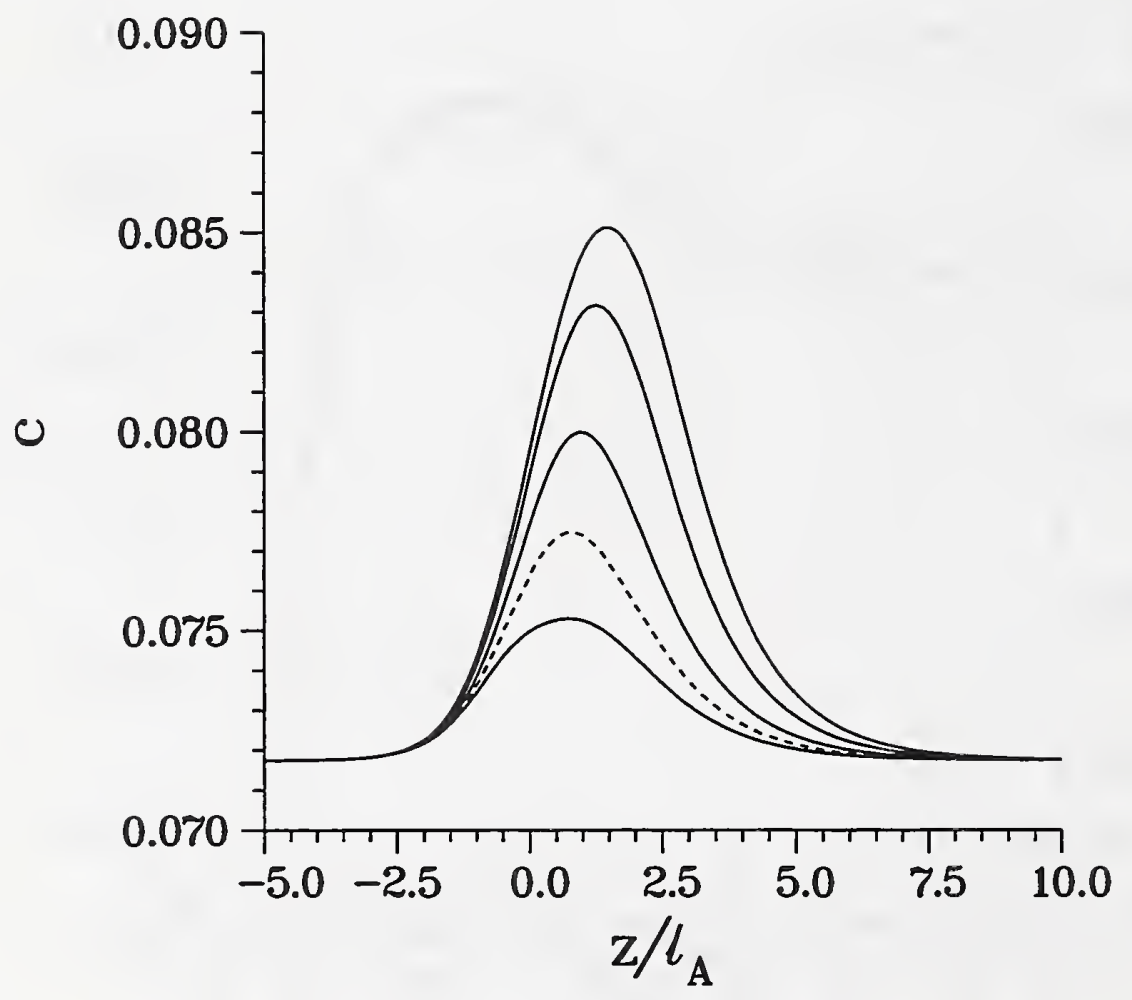

Figure 6: Solute profiles at a fixed growth velocity $V \ell_{A} / D_{L}=0.86$ for various values of the interface diffusivity. The diffusivity is given by Eq. (52) with $r(\phi)=\phi$ and $s(\phi)=16 \phi^{2}(1-\phi)^{2}$. The ratio of bulk diffusivities is fixed at $D_{L} / D_{S}=1$. The values of the ratio $D_{I} / D_{L}$ are, from the top curve to the bottom curve, $D_{I} / D_{L}=10,5,2,1$ (dashed curve), and $1 / 2$, respectively. 


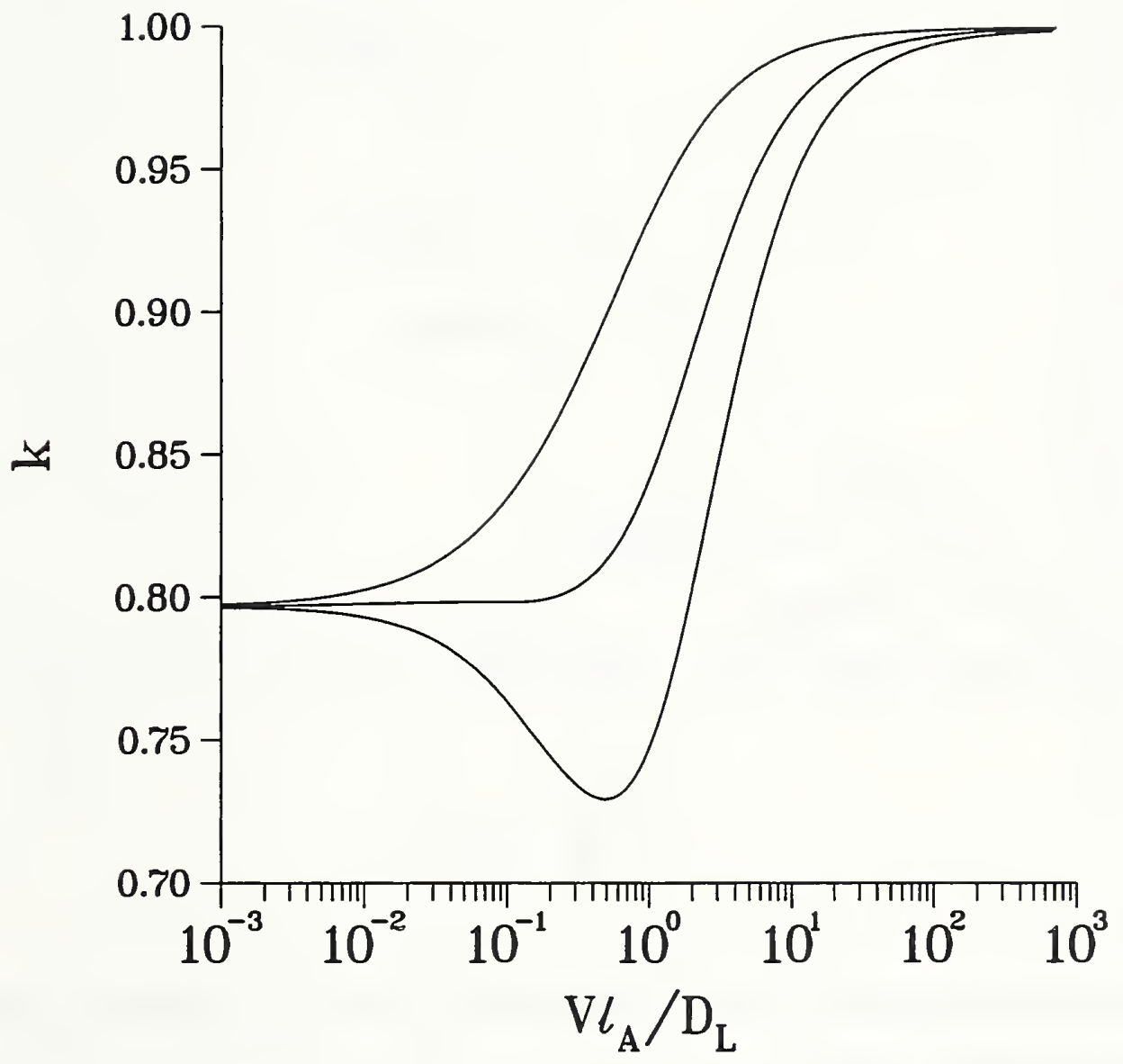

Figure 7: The partition coefficient $k=c_{\infty} / c_{\max }$ versus the normalized interface velocity $V \ell_{A} / D_{L}$ obtained from our numerical computations for various ratios of the surface tensions $\sigma_{B} / \sigma_{A}$ with $D_{S}=$ $D_{L}=10^{-5} \mathrm{~cm}^{2} \mathrm{~s}^{-1}$. From top to bottom the curves have $\sigma_{B} / \sigma_{A}=1, \sigma_{B} / \sigma_{A}=3$, and $\sigma_{B} / \sigma_{A}=4$, respectively. 


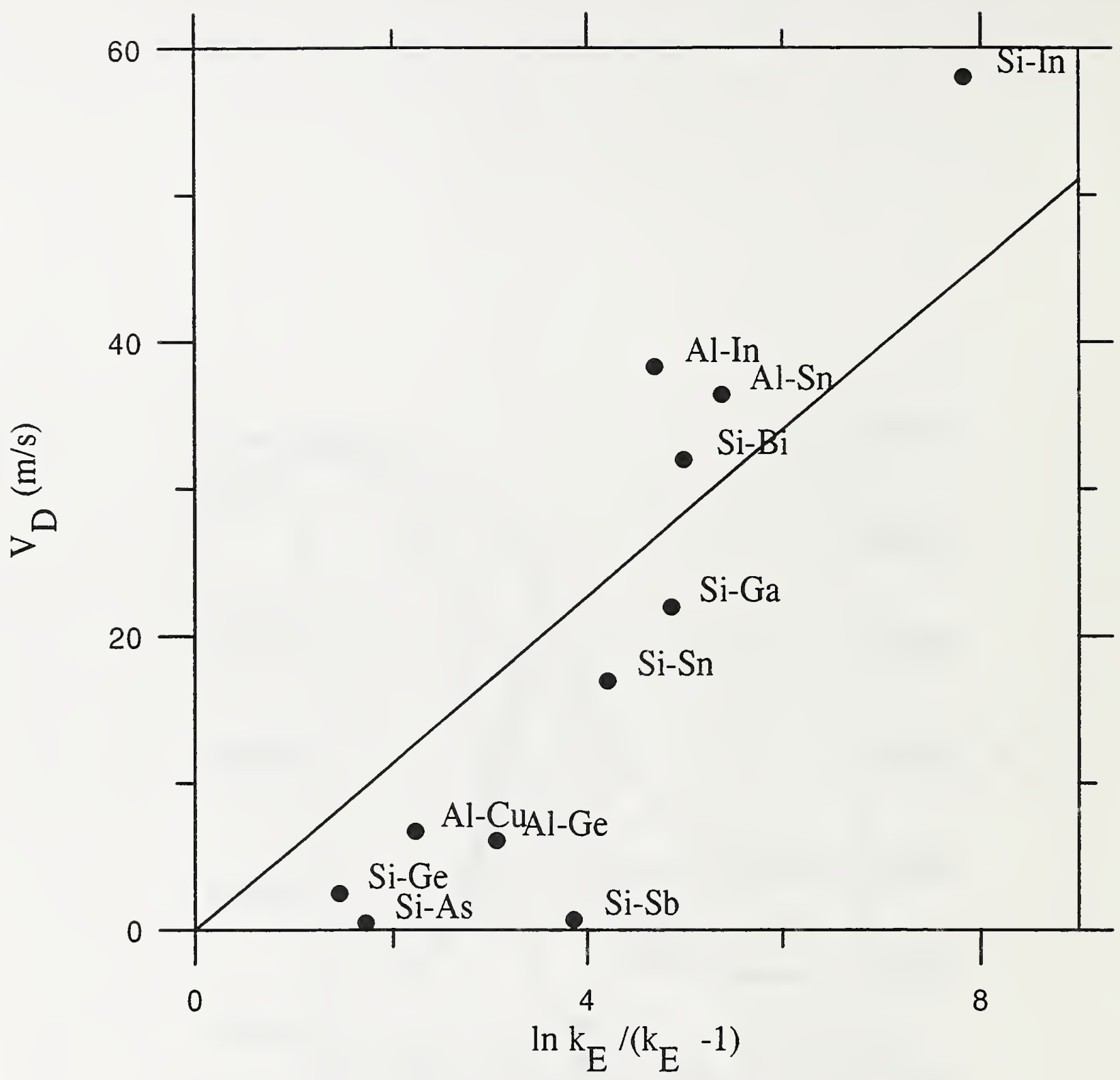

Figure 8: Experimental values for $V_{D}$ (see Aziz [58]) plotted versus the quantity $\ln k_{E} /\left(k_{E}-1\right)$. The line is a linear fit through the origin. 


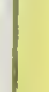


